ARTICLE

\title{
Sterols are required for the coordinated assembly of lipid droplets in developing seeds
}

Linhui Yu', Jilian Fan (10 ${ }^{1}$, Chao Zhou ${ }^{1} \&$ Changcheng Xu (i) ${ }^{1 凶}$

Lipid droplets (LDs) are intracellular organelles critical for energy storage and lipid metabolism. They are typically composed of an oil core coated by a monolayer of phospholipids and proteins such as oleosins. The mechanistic details of LD biogenesis remain poorly defined. However, emerging evidence suggest that their formation is a spatiotemporally regulated process, occurring at specific sites of the endoplasmic reticulum defined by a specific set of lipids and proteins. Here, we show that sterols are required for formation of oleosin-coated LDs in Arabidopsis. Analysis of sterol pathway mutants revealed that deficiency in several $\Delta^{5}$-sterols accounts for the phenotype. Importantly, mutants deficient in these sterols also display reduced LD number, increased LD size and reduced oil content in seeds. Collectively, our data reveal a role of sterols in coordinating the synthesis of oil and oleosins and their assembly into LDs, highlighting the importance of membrane lipids in regulating LD biogenesis.

\footnotetext{
${ }^{1}$ Biology Department, Brookhaven National Laboratory, Upton, NY 11973, USA. ${ }^{凶}$ email: cxu@bnl.gov
} 
S terols are isoprenoid-derived lipids with essential roles in cell structure, function, and physiology. As important components of biological membranes, sterols interact with phospholipids and proteins within the membrane, thereby regulating membrane fluidity, permeability, and membrane protein functions ${ }^{1-3}$. In addition to their structural role, sterols serve as a metabolic precursor to brassinosteroids (BRs), a group of steroid hormones that regulate a wide range of biological process 4 . Moreover, some sterols and sterol biosynthetic intermediates have been suggested to function as signaling molecules influencing transcription and signaling transduction pathways independent of BRs $s^{5,6}$. Furthermore, sterols are crucial players at the subcellular level in defining lipid rafts, sterol- and sphingolipidenriched functional membrane microdomains that are present in diverse organisms ${ }^{7}$. Such structures have been implicated in numerous biological processes as diverse as immunity in plants ${ }^{8}$, the synthesis of phospholipids in mammals ${ }^{9}$ and the biogenesis of lipid droplets $(\mathrm{LDs})^{10,11}$, specialized subcellular organelles for energy storage and lipid metabolism in all eukaryotes.

Plant sterols are synthesized in the endoplasmic reticulum (ER) from acetyl-CoA via the mevalonate pathway leading to the formation of cycloartenol (Supplementary Fig. 1), the first parental sterol composed of a tetracyclic ring nucleus with a free hydroxyl group and side chain at the C-3 or C-24 carbon atom, respectively. Cycloartenol is the common substrate for the synthesis of cholesterol, a minor sterol in plants, and of the major sterols, sitosterol, stigmasterol, and campesterol. The conversion of cycloartenol into major plant sterols involves a series of enzymatic reactions including methylation, demethylation, reduction, isomerization, and desaturation ${ }^{2,12}$. Among these enzymes, sterol methyltransferase $2 / 3 \quad(\mathrm{SMT} 2 / 3)$ and sterol methyl oxidase 2 (SMO2) define the second branch point in sterol biosynthesis, which directs precursor flow towards two parallel pathways leading to the generation of sitosterol and stigmasterol, two 24-ethyl-sterols or campesterol, a 24-methyl-sterol, respectively by both unique and shared enzymes, with the latter, including DWARF1 (DWF1), DWF5 and DWF7. Campesterol is the precursor to BRs. Consequently, disruption of the shared enzymatic steps downstream of SMT2/3 and SMO2 in the sterol biosynthetic pathway often leads to campesterol depletion, causing typical phenotypes of BR-deficient mutants such as severe dwarfism, impaired fertility, and yield. In addition to their free forms, sterols can be esterified with fatty acids to produce sterol esters (SEs) by sterol ester synthases, including phospholipid sterol acyltransferase1 (PSAT1) in Arabidopsis ${ }^{13}$ and the resulting SEs are stored in $\mathrm{LDs}^{2}$.

The physical structure of the LD consists of a hydrophobic core of SEs and triacyclglycerols (TAGs) surrounded by a monolayer of phospholipids and free sterols with a small set of proteins embedded or peripherally associated. Currently, the exact mechanistic details underlying LD biogenesis remain poorly understood $^{14,15}$. The most widely accepted model proposes that LDs originate from the specific ER subdomains defined by a specific set of lipids such as diacylglycerol (DAG) and proteins ${ }^{16-18}$, and is driven by ER membrane curvature, tension, and asymmetry 19,20. Several ER membrane proteins have been implicated in LD biogenesis in yeast and animals, likely via their effects on local lipid composition and hence ER membrane environments at sites of LD formation ${ }^{21-23}$. Furthermore, abundant LD structural proteins such as oleosins, have been shown to play a role in LD formation by sequestering neutral lipids from the ER bilayer into $\mathrm{LDs}^{24}$.

Oleosin possesses a long hydrophobic hairpin flanked by short amphipathic N- and C-terminal regions. On a LD, the hydrophobic hairpin is predicted to penetrate the monolayer into the neutral lipid core, whereas the flanking sequences interact with the monolayer membrane lipids on the LD surface ${ }^{25}$. During LD formation, oleosins are cotranslationally inserted into the ER, from where they then move to LDs. The Arabidopsis genome contains 17 oleosin genes specifically expressed in seeds and flower organs. Gene knockout or downregulation studies have revealed a key role of oleosins in regulating the size and stability of LDs in seeds ${ }^{26,27}$, but the precise role of oleosins in LD biogenesis and dynamics remains largely unknown.

Studies in animal model systems have shown that sterol synthesis, TAG metabolism, LD accumulation, and phospholipid synthesis are coregulated by a family of transcription factors, sterol regulatory element-binding proteins to maintain intracellular lipid homeostasis ${ }^{28}$. In plants, genes encoding key regulatory enzymes in sterol biosynthetic pathways show similar expression patterns to those in fatty acid and TAG synthesis during embryo development (Supplementary Fig. 2). In addition, several enzymes involved in sterol synthesis have been shown to be coordinately regulated during oil-seed development ${ }^{29}$ and the accumulation of high levels of free sterols precede the accumulation of TAGs in oilseeds ${ }^{29,30}$, but the functional role of sterols in TAG storage has not been examined in plants.

To understand the mechanisms and factors controlling LD biogenesis, we carried out a forward genetic screen using a transgenic line constitutively overproducing a green fluorescent protein (GFP)-tagged OLEOSIN1 fusion protein (OLE1) ${ }^{31}$ and searched for mutants with the altered size, number, and morphology of leaf LDs. We report here the identification and characterization of one of the mutants, M1-7, that is severely defective in the accumulation of LDs in leaves. We showed that altered membrane sterol composition, but not deficiency in BRs and SEs compromises LD accumulation in leaves and seeds. The possible implication of this finding in our understanding of the role of sterols in LD formation is discussed.

\section{Results}

Isolation of LD mutants. Seeds of the homozygous Arabidopsis transgenic line overexpressing OLE1 driven by the Cauliflower Mosaic Virus (CaMV) promoter ${ }^{31}$ were mutagenized with ethyl methanesulfonate. To identify mutants defective in TAG accumulation, leaf samples from individual $\mathrm{M}_{2}$ plants were examined under a fluorescence microscope. Among 10 putative mutants isolated thus far, one of the mutants, designated M1-7, was almost completely devoid of leaf LDs and TAG. Mutant plants grown on soil displayed dark-green leaves and a short, robust statute (Fig. 1a), and contained only a few LDs and very limited amounts of TAG in young and mature leaves, though both the TAG content and LD size were slightly increased as leaves aged (Fig. 1b-d). This mutant was subsequently backcrossed to the parental line. The self-pollinated $\mathrm{F}_{1}$ plants produced $\mathrm{F}_{2}$ progeny that segregated for dwarf plants in a ratio of $\sim 4-1$ (48 out of 205 , $23.4 \%$ ), and all the 48 plants had drastically reduced LD accumulation in leaves. These results suggest that LD deficiency and the dwarf phenotype are tightly linked and cosegregate as a single recessive trait.

The M1-7 mutant represents a new allele of $\boldsymbol{d w f 5}$. To identify the mutation responsible for the LD-deficient phenotype, the M1-7 mutant was outcrossed to ecotype Landsberg erecta to generate a mapping population that was used for rough mapping, and the mutant locus was delimited to an $\sim 1500-\mathrm{kb}$ region (Fig. $2 \mathrm{a}$ ). Whole genomic DNA sequencing identified six GC:AT transitions that generated nonsense or missense mutations in protein-coding sequences in this region (Supplementary data 1). Among them is a $\mathrm{G}$ to A nonsense transition in the 11th exon of the gene At1g50430, 
a

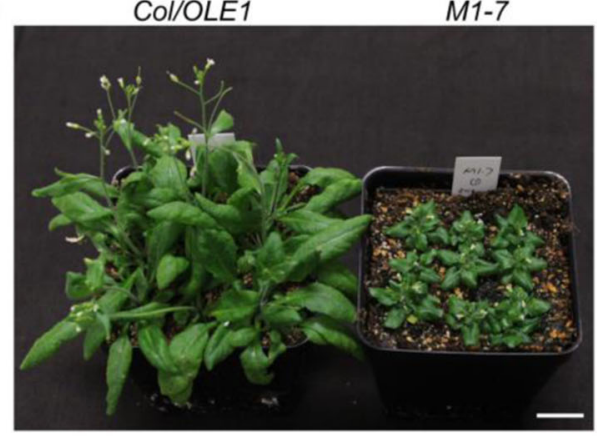

\author{
C
}

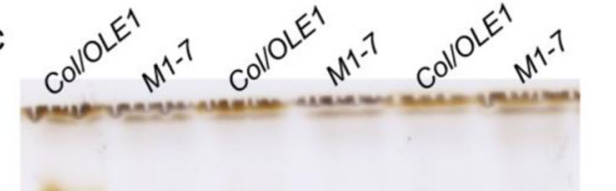

TAG

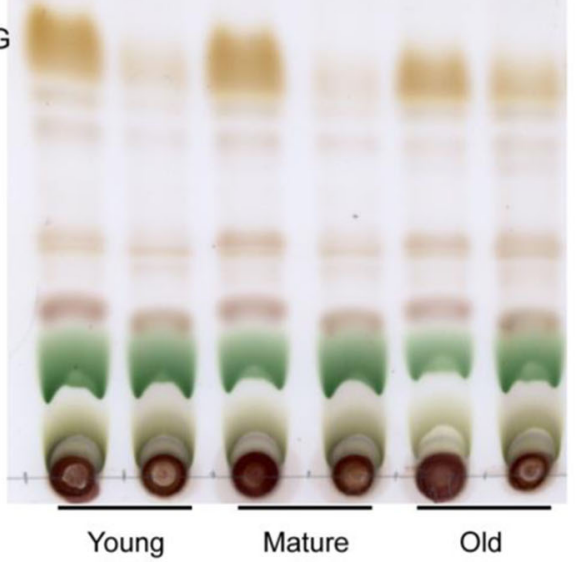

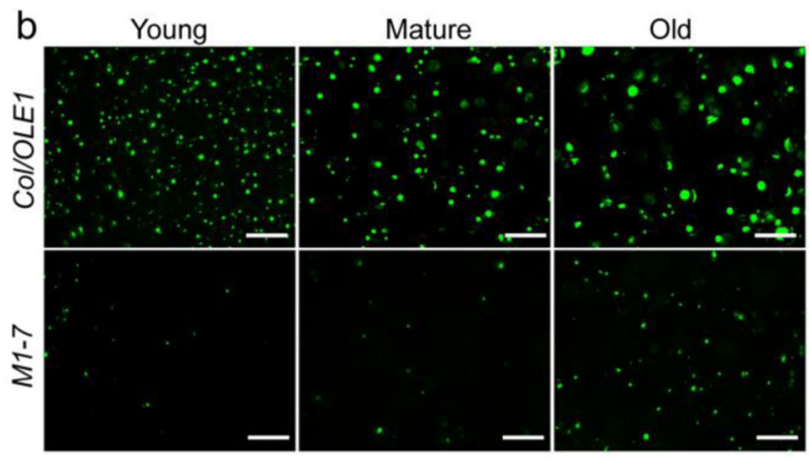

d

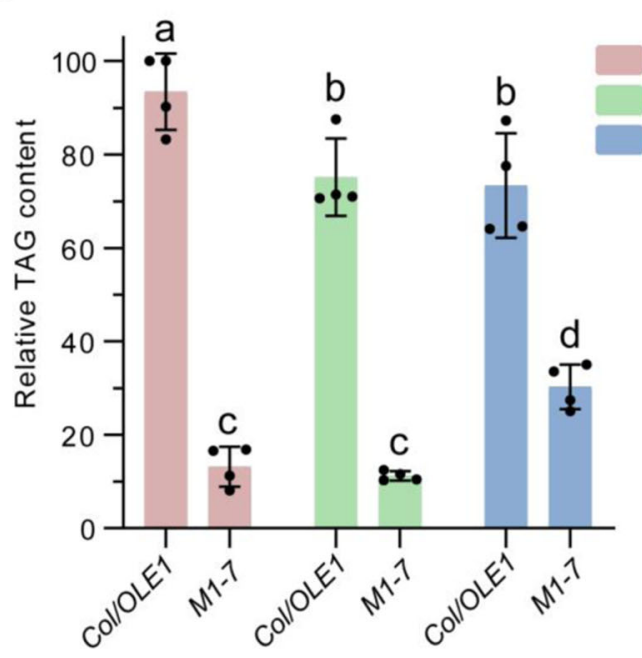

Young

Mature

Old

Fig. 1 TAG content and LD abundance are dramatically decreased in M1-7 mutant. a Phenotypes of 4-week-old M1-7 and Col/OLE1. Bar $=2 \mathrm{~cm}$. b Representative images of LDs (OLE1-GFP, green) in rosette leaves of different ages of 4-week-old plants (young leaves: the upper growing leaves; mature leaves: the newest expanded leaves; old leaves: the oldest expanded green leaves). The experiment was repeated 5 times with similar results. Bar $=50 \mu$ m. c Thin-layer chromatogram of neutral lipids in leaves of M1-7 and Col/OLE1. Lipids were visualized with 5\% sulfuric acid by charring. d Relative TAG contents in leaf of M1-7 and Col/OLE1 quantified by Image J software. Data are mean \pm SD. of four biological replicates. Different letters indicate significant differences at $P<0.05$, as determined by one-way ANOVA with Tukey's multiple comparisons test.

which encodes DWF5, a sterol $\Delta^{7}$-sterol-C5-desaturase. This mutation introduces a premature stop codon that is expected to lead to a truncated protein lacking 76 amino acids at the C-terminus, and the M1-7 mutant was hence renamed as $d w f 5-10 / O L E 1$ (Fig. 2b). To confirm that DWF5 is the causative gene for the LDdeficient phenotype, we isolated a homozygous dwarf mutant with a T-DNA insertion in the 8th intron of the gene and designated it $d w f 5-8$ (Fig. 2a, b). This T-DNA mutant, when introduced into the OLE1 line, recapitulated the neutral lipid storage phenotype of $d w f 5-10 / O L E 1$ (Fig. 2c-e). In addition, introducing the DWF5 coding sequence driven with CaMV35S promoter into $d w f 5-10 /$ OLE1 led to increases in plant growth (Supplementary Fig. 3a, b) and LD abundance (Supplementary Fig. 3c).

The role of sterols in neutral lipid storage is BR-independent. To exclude the possibility that the LD-deficient phenotype was due to a lack of BRs or block of BR signaling, we isolated T-DNA insertion mutants in DWF4 and Brassinosteroid-Insensitive 1 (BRI1), which encodes an enzyme necessary for BR synthesis (Supplementary Fig. 1) and the BR receptor, respectively ${ }^{4}$. These two mutants, when introduced into the OLE1 line, caused no obvious changes in leaf LD abundance and TAG content (Supplementary Fig. 4a, b). In addition, external feeding of 24-epibrassinolide (24-epiBL) failed to rescue the LD deficiency in $d w f 5-8 / O L E 1$ and $d w f 7-4 / O L E 1$ (Supplementary Fig. $4 c$, d). Moreover, blocking endogenous BR biosynthesis by brassinazole (BRZ) or applying exogenous 24-epiBL has no effect on TAG content in shoots (Supplementary Fig. 4e). These data suggest that the LD- and TAG-deficient phenotype is not due to defects in BR synthesis or BR signaling transduction.

Deficiency in 24-ethyl/ethylidene- $\Delta^{5}$-sterols results in decreases in leaf TAG accumulation in the OLE1 line. Sterol analysis showed that total free sterols decreased by approximately $74 \%$ in $d w f 5$ mutants compared with the wild type (WT), due primarily to drastic decreases in the major sterols, such as sitosterol, campesterol, stigmasterol, and isofucosterol (24-ethylidene- $\Delta^{5}$-sterol) (Supplementary Table 1). In addition, there were increases in levels of several unusual sterol molecules, such as stigmasta5,7,22-trienol and stigmasta-5,7-dienol in $d w f 5$ mutants. To gain further insight into whether and how changes in sterol profiles impact TAG storage, we isolated several additional T-DNA insertion mutants defective in various steps of the sterol biosynthesis pathway and introduced these mutations into the OLE1 line by genetic crossing. The $d w f 1-2$ mutant is defective in the conversion of 24-methylenecholesterol and isofucosterol to campesterol and sitosterol, respectively ${ }^{32}$. Consequently, this mutant lacks sitosterol and campesterol, but instead accumulates 


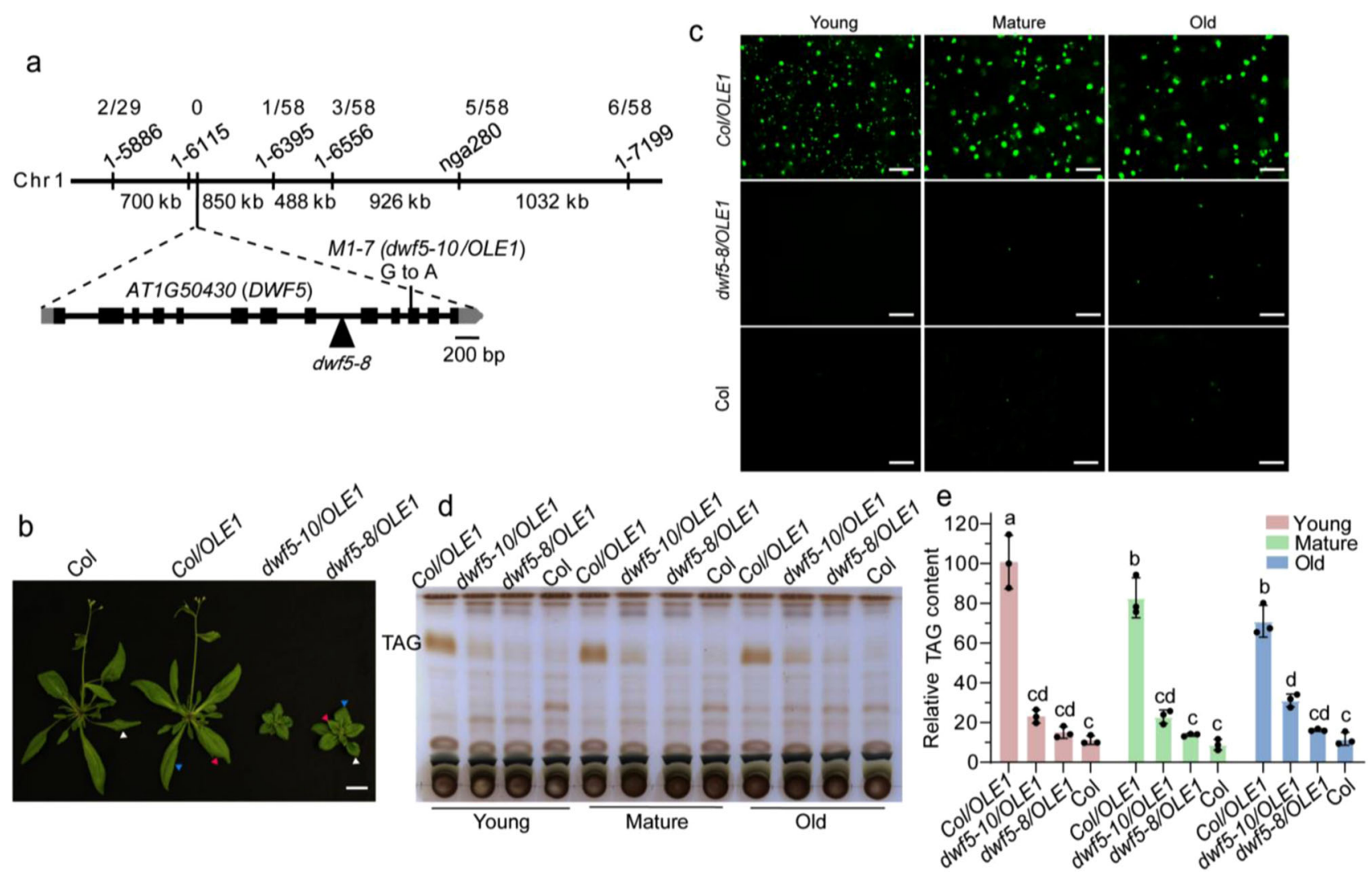

Fig. 2 Disruption of DWF5 results in defects in TAG and LD accumulation in leaves. a The map position of the M1-7 mutation and structure of DWF5 (At1g50430). Markers used for mapping and the respective number of recombinants are indicated. The exons of DWF5 are shown as black boxes and introns are indicated by lines. The point mutation in the $d w f 5$ mutant allele is indicated with a line and the T-DNA insertion with a triangle. $\mathbf{b}$ Growth phenotypes of 4-week-old plants. Red, blue, and white arrowheads indicate young, mature, and old leaves (for definitions, see Fig. 1), respectively. Bar $=2 \mathrm{~cm}$. c Representative images of LDs (OLE1-GFP, green) in leaves of 4-week-old Col/OLE1 and dwf5-8/OLE1 plants and LDs in wild-type leaves stained by Nile Red. The experiment was repeated 4 times with similar results. Bar $=50 \mu \mathrm{m}$. d, e, TLC analysis of neutral lipids. Lipids were extracted from leaves of 4-week-old plants and visualized with $5 \%$ sulfuric acid by charring (d). Relative contents of TAG were quantified by Image J (e). Data are mean \pm SD of three biological replicates. Different letters indicate significant difference at $P<0.05$, as determined by one-way ANOVA with Tukey's multiple comparisons test.

their immediate precursors without major changes in total free sterol content (Supplementary Table 1). The $d w f 7-4$ mutant is defective in the $\Delta^{7}$-sterol-C5-desaturase ${ }^{32}$. This mutant contains very limited amounts of all major sterols but accumulate an unusual sterol molecule $\Delta^{7}$ - sitosterol and the total free sterol content is decreased slightly in $d w f 7-4$ compared with WT (Supplementary Table 1). The cvp1-3 smt3-1 double mutant contains a fivefold higher level of campesterol, a 24-methyl- $\Delta^{5}$ sterol, while the amount of 24 -ethyl- $\Delta^{5}$-sterols is reduced by $63 \%$ and total free sterol content remains largely unaltered (Supplementary Table 1$)$. Introducing the $d w f 1-2$ into the OLE1 line resulted in no significant change of TAG content in leaves. In contrast, disruption of DWF7 or SMT2/3 caused drastic decreases in TAG accumulation in the OLE1 line (Fig. 3). Together, these results suggest that deficiency in 24-ethyl- $\Delta^{5}$-sterols and 24 ethylidene- $\Delta^{5}$-sterol is responsible for reduced TAG and LD accumulation in sterol mutants.

In addition to the changes in free sterol content and composition, all the sterol mutants tested, particularly $d w f 5$, $d w f 7$, and $c v p 1-3$ smt3-1 displayed significantly reduced levels of SEs (Supplementary Table 2). To test whether the deficiency in SEs affect TAG accumulation, we obtained T-DNA insertion mutants disrupted in PSAT1 and crossed the mutants with the OLE1 line. As expected, disruption of PSAT1 caused major reductions in SE content in leaves (Supplementary Table 2). However, there were no significant changes in LD abundance and
TAG content in leaves and mature seeds of psat1/OLE1 mutants compared with those of the OLE1 line (Supplementary Fig. 5). Together, these results suggest that the decreased SE levels are not responsible for the LD-deficient phenotype in the sterol mutants.

The OLE1 transcript level correlates strongly with LD abundance. To test whether sterols directly impact $L D$ abundance via their effects on OLE1 expression, we analyzed the OLE1 transcript level in $d w f$ mutants, two additional LD-deficient mutants (M2-1 and M3-1) isolated from the same genetic screen as for M1-7 and the phospholipid:diacylglycerol acyltransgerase1 (PDAT1) knockout line carrying the OLE1 transgene (pdat1-2/OLE1). Unlike sterol-deficient $d w f$ mutants, M2-1, M3-1, and pdat1-2/ OLE1 showed normal growth phenotypes and thus presumably contained normal levels of sterols. However, like $d w f 5$ and $d w f 7$, all these three LD-deficient mutants had drastic decreases in levels of the OLE1 transcript and there is a strong positive correlation between the OLE1 transcript levels and LD abundance in leaves (Supplementary Fig. 6). These results suggest that the expression level of the OLE1 transgene is related to $\mathrm{LD}$ abundance rather than to amounts of sterols per se.

TAG metabolism is not altered in $d w f 5$ and $d w f 7$ mutants lacking OLE1. Mutants defective in DWF1, DWF4, DWF5, and DWF7 are all dwarf. Their morphology, growth, and 

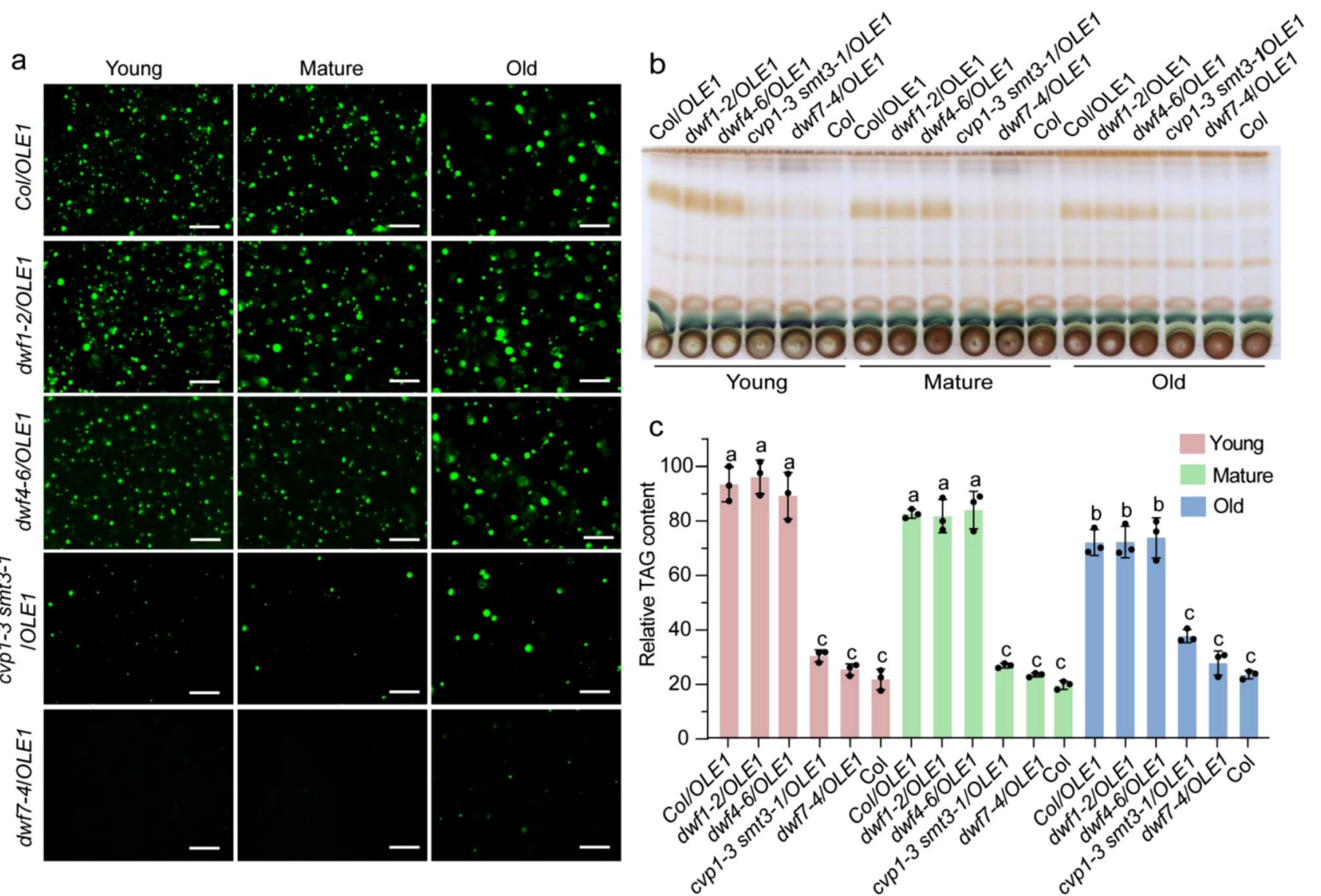

Fig. 3 Disruption of DWF7 or SMT2/3 but not DWF1 and DWF4 results in defects in TAG and LD accumulation in leaves. a Representative images of LDs (OLE1-GFP, green) in young, mature, and old (for definitions, see Fig. 1) leaves of 4-week-old plants. The experiment was repeated four times with similar results. Bar $=50 \mu \mathrm{m}$. b, c TLC analysis of neutral lipids. Lipids were isolated from leaves of 4 -week-old plants and visualized with $5 \%$ sulfuric acid by charring (b). Relative contents of TAG were quantified by Image J (c). Data are mean \pm SD of three biological replicates. Different letters indicate significant differences at $P<0.05$, as determined by one-way ANOVA with Tukey's multiple comparisons test.

developmental patterns are similar among the mutants but quite different compared with WT (Supplementary Fig. 7). Therefore, to address the question of how the altered sterol profile affects TAG metabolism in the sterol mutants, we first compared rates of TAG synthesis and turnover among $d w f 4-6, d w f 5-8$, and $d w f 7-4$ mutants with or without the OLE1 transgene in pulse-chase experiments with cold or radiolabeled oleic acid (18:1). TAG content in leaves of $d w f 4-6, d w f 5-8$, and $d w f 7-4$ was very low prior to feeding (Fig. 4a). Feeding with $0.5 \mathrm{mM} 18: 1$ or $0.02 \mathrm{mM}{ }^{14} \mathrm{C}$ 18:1 led to large increases in TAG content and radiolabeled TAG in all three $d w f$ mutants. During the 24 -h chase, TAG content and radiolabeled TAG decreased by $50 \%$ and more than $80 \%$, respectively, in all the mutants lacking the OLE1 transgene (Fig. $4 \mathrm{a}, \mathrm{b}$ ). The higher turnover rate of radiolabeled TAG than that of cold TAG may reflect less TAG accumulation in pulsechase experiments with ${ }^{14} \mathrm{C}-18: 1$ than with cold 18:1 since the concentration of the later is 25 -fold higher than that of the former. Nevertheless, there were no significant differences in TAG levels either following the pulse or during the chase between $d w f 4-6$ and $d w f 5-8$ or $d w f 7-4$, except that $d w f 5-8$ appeared to contain slightly higher amounts of radiolabeled TAG following the pulse with ${ }^{14} \mathrm{C}-18: 1$. These results suggest that disruption of DWF5 or DWF7 has no major impact on TAG metabolism in leaves.

To further investigate lipid metabolism in the sterol mutants, we carried out ${ }^{14} \mathrm{C}$-acetate pulse-chase experiments using detached leaves. The rates of labeled acetate incorporation into total fatty acids, as well as into TAG were similar between $d w f 1 / 4$ and $d w f 5 / 7$ (Supplementary Fig. 8a, b). Arabidopsis PDAT1 plays a critical role in TAG synthesis in leaves ${ }^{31}$. Assays for PDAT activity in microsomal membranes revealed that sterols had no effect on TAG formation from ${ }^{14} \mathrm{C}$-labeled phosphatidylcholine (PC) in leaves (Supplementary Fig. 8d). During the chase, the label in TAG decreased in all the sterol mutants with no significant differences between $d w f 1 / 4$ and $d w f 5 / 7$, reflecting a similar TAG degradation rate in these sterol mutants (Supplementary Fig. 8c). Together, these results suggest that deficiency in 24-ethyl- $\Delta^{5}$-sterols and 24-ethylidene- $\Delta 5$-sterol in the mutants lacking OLE1 has no effects on rates of fatty acid synthesis, TAG synthesis, and turnover.

Leaf TAG turnover is accelerated in $d w f 5 / O L E 1$ and $d w f 7 /$ OLE1. In the presence of the OLE1 transgene, $d w f 4-6$ leaves contained a much higher amount of TAG than leaves of $d w f 5-8$ or $d w f 7-4$ following the incubation with $0.5 \mathrm{mM} 18: 1$, likely due to a higher amount of preexisting TAG accumulation in $d w f 4-6 / O L E 1$ than in $d w f 5-8 / O L E 1$ and $d w f 7-4 / O L E 1$ (Fig. 4a). In line with this possibility, the amount of radiolabeled TAG in $d w f 4-6 / O L E 1$ following the pulse with ${ }^{14} \mathrm{C}-18: 1$ was similar to that in $d w f 5-8 /$ $O L E 1$ and $d w f 7-4 / O L E 1$, suggesting again that disruption of DWF5 or DWF7 has no impact on the rate of TAG synthesis (Fig. 4b). During the 24-h chase, TAG levels decreased slightly in $d w f 1-2 / O L E 1$ and $d w f 4-6 / O L E 1$ but markedly in $d w f 5-8 / O L E 1$ and $d w f 7-4 / O L E 1$ (Fig. 4a, b, Supplementary Fig. 9). Like the situation in $d w f$ mutants lacking OLE1, radiolabeled TAG accumulated following ${ }^{14} \mathrm{C}-18: 1$ feeding decreased faster than TAG 

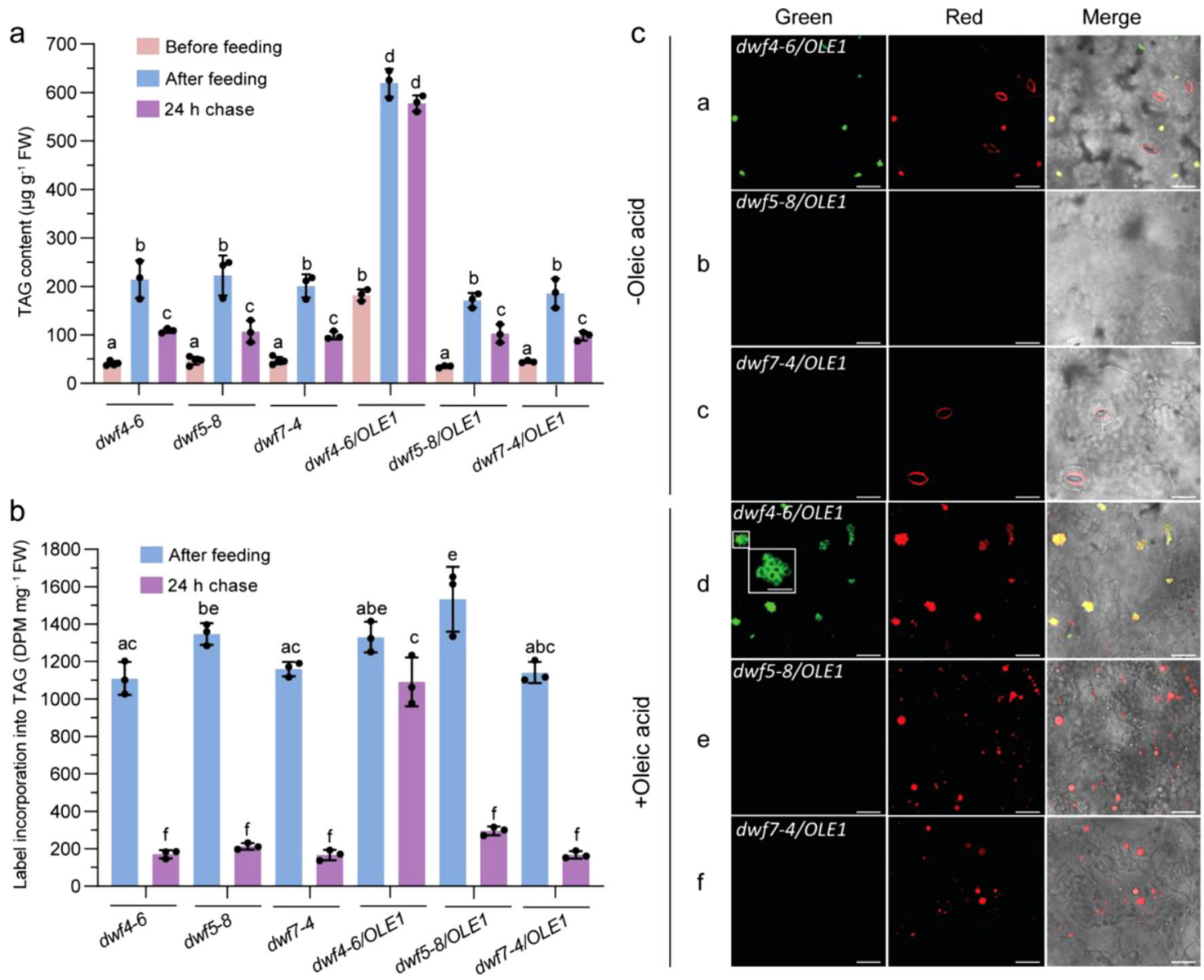

Fig. 4 TAG metabolism and LD accumulation in leaves of $\boldsymbol{d w}$ mutants as revealed by oleic acid feeding. a TAG contents in leaves before oleic acid feeding, after $0.5 \mathrm{mM}$ cold oleic acid feeding for overnight and after $24 \mathrm{~h}$ of chase. Lipids were extracted from leaves and quantified by GC-MS. Four biological replicates were performed for $d w f 4-6$, dwf5-8, and $d w f 7-4$ before feeding while the others had three biological replicates. $\mathbf{b}$ Label incorporation into TAG in leaves after ${ }^{14} \mathrm{C}$-oleic acid feeding and after $24 \mathrm{~h}$ of chase. Lipids were extracted from leaves and separated by TLC. Radiolabeled TAGs were quantified by liquid scintillation counting. Three biological replicates were carried out for each sample. $\mathbf{c}$ LDs in leaves after $0.5 \mathrm{mM}$ cold oleic acid feeding for overnight. LDs were stained by BODIPY red and observed with a confocal scanning laser microscope. The experiment was repeated 3 times with similar results. Bars $=20 \mu \mathrm{m}$ ( $5 \mu \mathrm{m}$ in insert). In $\mathbf{a}$ and $\mathbf{b}$, data are mean \pm SD. Different letters indicate significant differences at $P<0.05$, as determined by oneway ANOVA with Tukey's multiple comparisons test.

accumulated following feeding with cold 18:1 in all $d w f / O L E 1$ mutants, particularly in $d w f 5-8 / O L E 1$ and $d w f 7-4 / O L E 1$, during the chase. Together, these results suggest that disruption of DWF5 or DWF7 accelerates TAG turnover in the OLE1 transgenic line.

Leaves of $d w f 5 / O L E 1$ and $d w f 7 / O L E$ mutants accumulate largesized individual LDs lacking oleosin upon oleic acid feeding. Overexpression of GFP-tagged OLE1 in Arabidopsis leaves resulted in the clustering of small $\mathrm{LDs}^{31}$. These OLE1-GFPdecorated LD clusters were clearly seen in leaves of $d w f 4-6 / O L E 1$ and were colocalized with LD clusters stained with a neutral lipid dye, BODIPY red, as expected (Fig. 4c, panel a). In contrast, both the green and red fluorescent signals were barely detectable in $d w f 5-8 / O L E 1$ and $d w f 7-4 / O L E 1$ leaves (Fig. $4 \mathrm{c}$, panel $\mathrm{b}$ and c). To test whether sterol deficiency affects LD organization and morphology, detached leaves of $d w f 4-6 / O L E 1, d w f 5-8 / O L E 1$, and $d w f 7-4 / O L E 1$ were first incubated with $0.5 \mathrm{mM}$ 18:1 overnight and then stained with BODIPY red. Consistent with increased TAG content, feeding with 18:1 resulted in an increase in the size and number of OLE1-GFP-decorated LD clusters in $d w f 4-6 / O L E 1$ and the vast majority of green fluorescent signals colocalized with BODIPY-stained LD clusters. The individual LD in the clusters was small with diameters of less than $1 \mu \mathrm{m}$ (Fig. 4c, panel d). Strikingly, even though 18:1 feeding led to the accumulation of large amounts of TAG (Fig. 4a), green fluorescent signals were barely detected in 18:1-fed $d w f 5-8 / O L E 1$ and $d w f 7-4 / O L E 1$ leaves (Fig. 4c, panel e and f). BODIPY staining, on the other hand, revealed the presence of abundant large-sized LDs with diameters of up to $10 \mu \mathrm{m}$, and most of them were present in solitary forms (Fig. 4c, panel e and f).

Mutants disrupted in DWF5 and DWF7 have reduced seed TAG content and altered seed morphology. The data presented thus far suggest that deficiency in 24-ethyl- $\Delta^{5}$-sterols and 24ethylidene- $\Delta^{5}$-sterol results in the formation of unstable LDs lacking OLE1 coating even in the presence of the constitutively expressed OLE1 transgene. The endogenous oleosin genes are not expressed in leaves but in developing seeds during seed oil accumulation. To exclude the possibility that the observed LD phenotypes in leaves of sterol mutants are due to artifacts arising from the ectopic expression of OLE1 or oleic acid feeding, we sought to investigate the role of sterols in oil accumulation in seeds of sterol mutants with or without the OLE1 transgene. To this end, we first analyzed sterol content and composition in seeds. Total free sterol levels in mature seeds were increased in 

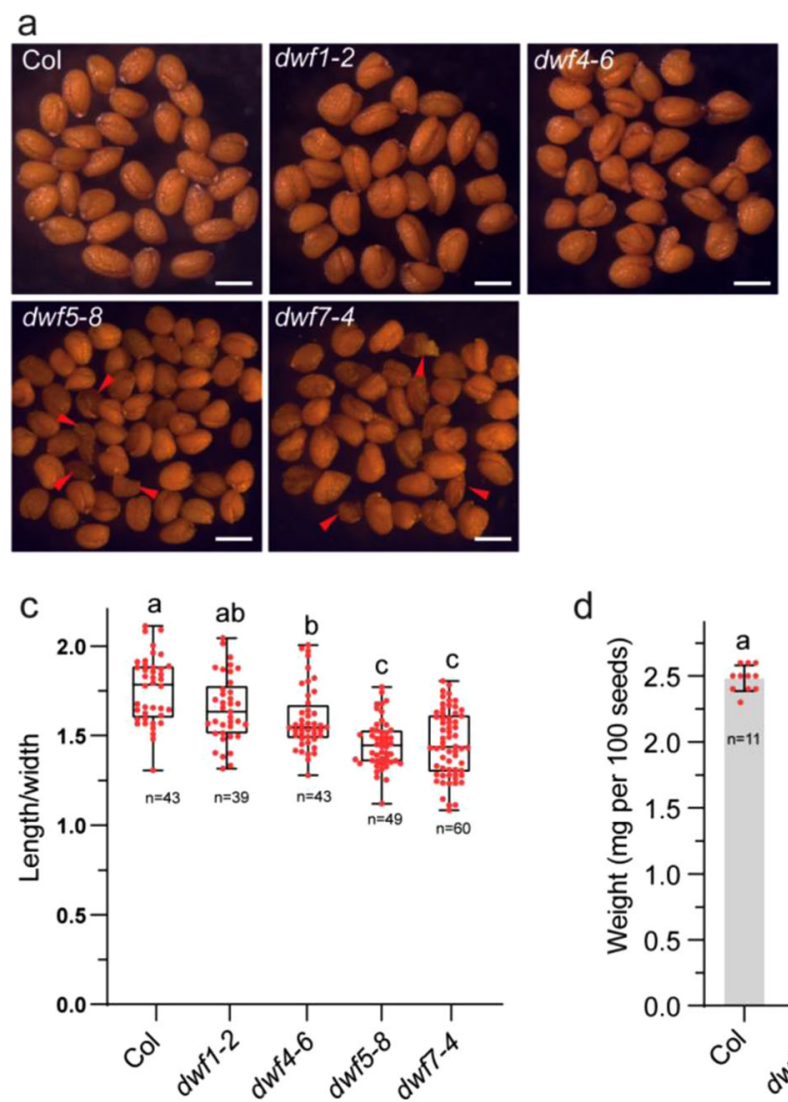

d

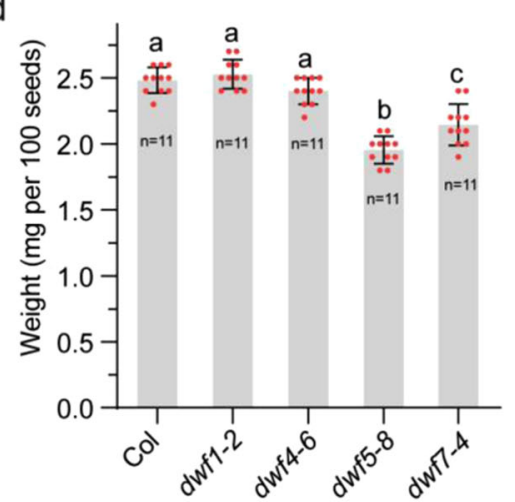

b

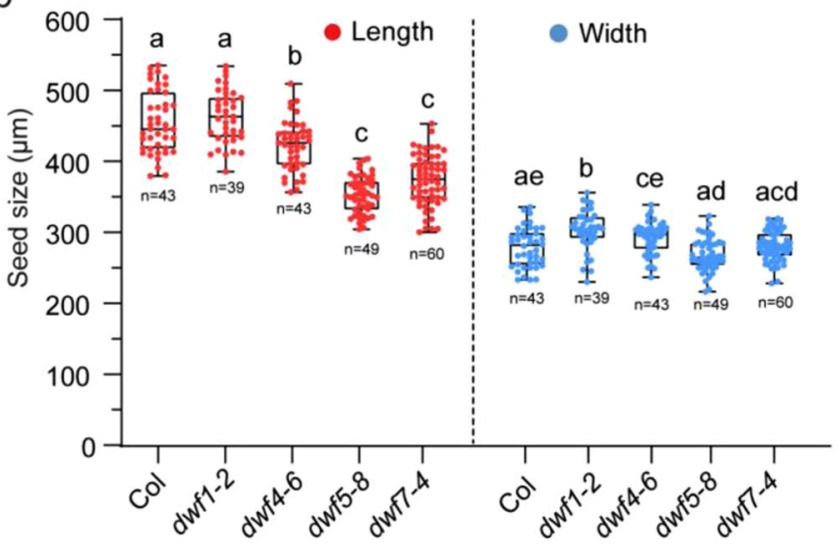

e

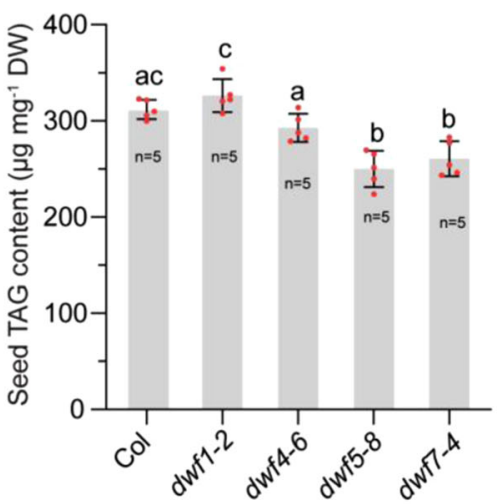

Fig. 5 Reduced seed TAG content and altered seed morphology in $\mathbf{d w f 5}$ and $\boldsymbol{d w f 7}$ mutants. a Representative phenotypes of dry seeds of wild type (Col), $d w f 1-2, d w f 4-6, d w f 5-8, d w f 7-4$. Red triangles indicate severe wrinkled seeds. Bar $=400 \mu$ m. b Seed size. c Length to width ratios of seeds. Numbers in $\mathbf{b}$ and $\mathbf{c}$ represent the number of seeds analyzed for data collection. d Weight per 100 dry seeds. e Seed TAG contents quantified by GC-MS. In $\mathbf{b}$ and $\mathbf{c}$, the box plot boundaries reflect the interquartile range, the center line is the median and the whiskers represent $1.5 \times$ the interquartile range from the lower and upper quartiles. Numbers in $\mathbf{b}$ and $\mathbf{c}$ represent the number of seeds analyzed for data collection. Data in $\mathbf{d}$ and $\mathbf{e}, n$ represent the number of biological replicates. Data are mean $\pm \mathrm{SD}$. In $\mathbf{b}, \mathbf{c}, \mathbf{d}$, and $\mathbf{e}$, different letters indicate significant differences at $P<0.05$, as determined by one-way ANOVA with

Tukey's multiple comparisons test.

psat1 mutants, remained largely unchanged in cvp1-3 smt3-1 and were decreased by 72,75 , and $56 \%$ in $d w f 5-8, d w f 5-10 / O L E 1$, and $d w f 7-4$, respectively, compared with the corresponding controls (Supplementary Table 3). Like what was found in leaves of $d w f 5$ and $d w f 7$ mutants (Supplementary Table 1), the decreases in total free sterols in mutant seeds were largely attributable to decreases in levels of 24-ethyl- $\Delta^{5}$-sterols including sitosterol and stigmasterol, whereas unusual sterols such as stigmasta-5,7,22-trienol and stigmasta-5,7-dienol in $d w f 5-8$ and $\Delta^{7}$-sitosterol in $d w f 7-4$ were increased (Supplementary Table 3 ).

We next examined the morphology of mature seeds of $d w f$ mutants. Both $d w f 5-8$ and $d w f 7-4$ seeds were smaller and rounder than WT seeds (Fig. 5a) and their length, width, and length/width ratios were decreased compared with WT and $d w f 1-2$ (Fig. 5b, c). In addition, seed weights of the $d w f 5-8$ and $d w f 7-4$ mutants were significantly decreased compared to the weight of WT seeds (Fig. 5d). Unlike the mutants upstream of 24-methylenelophenol, such as smt1 and hyd1, which commonly show defects in embryogenesis, downstream mutants $c v p 1 / s m t 2, d w f 7, d w f 5$, and $d w f 1 / d w f 1-2$ do not exhibit altered embryogenesis ${ }^{32}$. However, like what was found in $d w f 5-1$ seeds $^{33}$, a substantial proportion of seeds in $d w f 5-8$ and $d w f 7-4$ were dark brown in color and wrinkled to some extent (Fig. 5a). A possible reason of the seed phenotype is TAG reduction in these wrinkled seeds. Indeed, TAG content per milligram seeds decreased significantly by $17.6 \%$ and $11.6 \%$ in $d w f 5-8$ and $d w f 7-4$ seeds, respectively, compared with WT, whereas no significant change was found in $d w f 1-2$ and $d w f 4-6$ seeds (Fig. 5e). Seeds of $d w f 5-10 / O L E 1$ showed a much more severe wrinkled appearance than that of $d w f 5-8$ mutant, with about $37.4 \pm 2.4 \%$ seeds being affected in the former (Supplementary Fig. 10a-d). Consequently, TAG content in $d w f 5-10 / O L E 1$ decreased by up to $40 \%$ compared with Col/OLE1 seeds (Supplementary Fig. 10e). In addition, oil levels in developing embryos decreased by 73 and $57 \%$ at the bent cotyledon stage in $d w f 5-8$ and $d w f 7-4$, respectively and by 50 and $20 \%$ at the mature green stage in $d w f 5-8$ and $d w f 7-4$, respectively (Supplementary Fig. 11).

Disruption of DWF5 or DWF7 results in an increase in LD size in seeds. To investigate the effect of sterol deficiency on LD morphology, we carried out the microscopic analysis of LDs in embryos from mature seeds. Transmission electron microscopy (TEM) imaging revealed that LDs in $d w f 1-2$ seed cells showed a similar size distribution pattern to that of the WT. However, LDs in $d w f 5-8$ mutants were more heterogeneous, larger, and rounder compared with LDs in WT and $d w f 1-2$ (Fig. 6a). Quantitative analysis revealed that both the length of long axis and short axis of LDs in the $d w f 5-8$ mutant were significantly increased while the ratio of the length of long axis to short axis was significantly decreased compared with LDs in WT seeds (Fig. 6b, c). Consequently, the LD number per microscopical field was significantly decreased in $d w f 5-8$ seeds (Fig. $6 \mathrm{~d}$ ). LDs in $d w f 7-4$ seeds were also enlarged compared with those in WT 
a

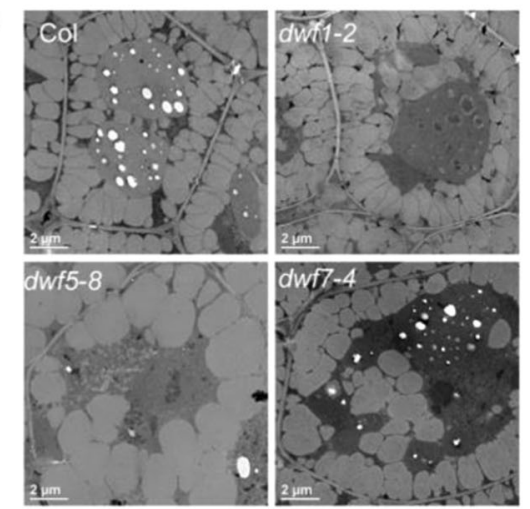

C

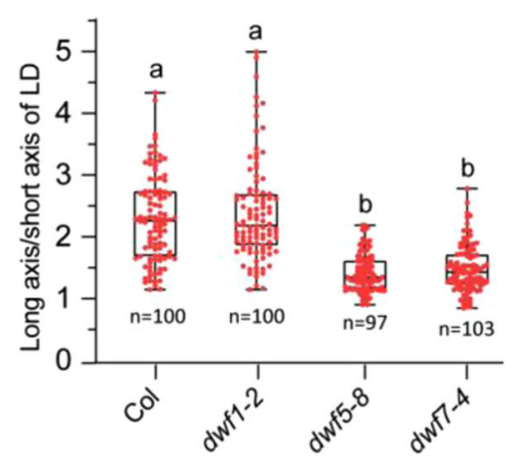

e
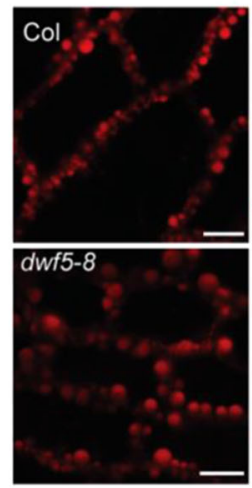

g

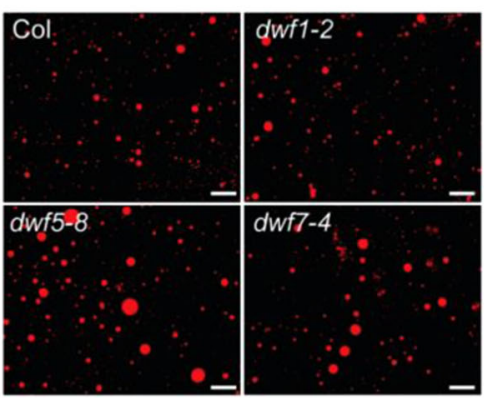

b

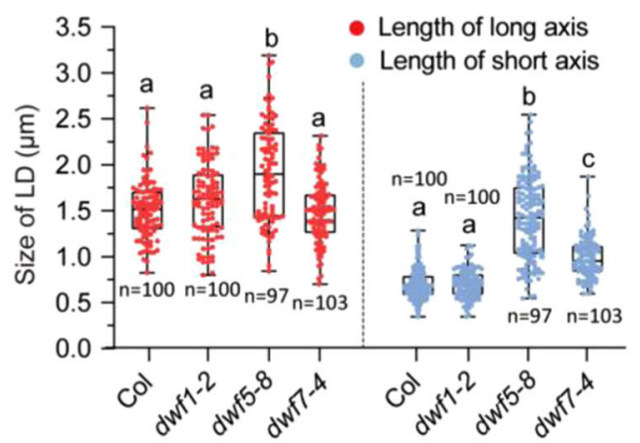

d

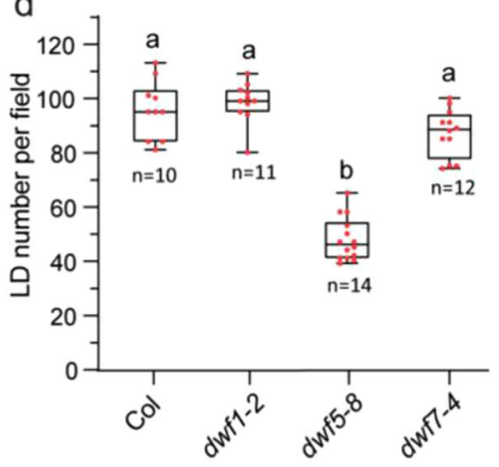

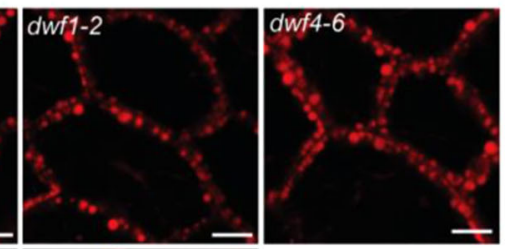

$d w 77-4$

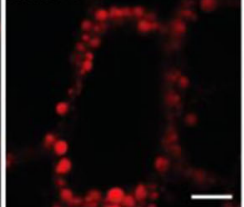

h 1 a a
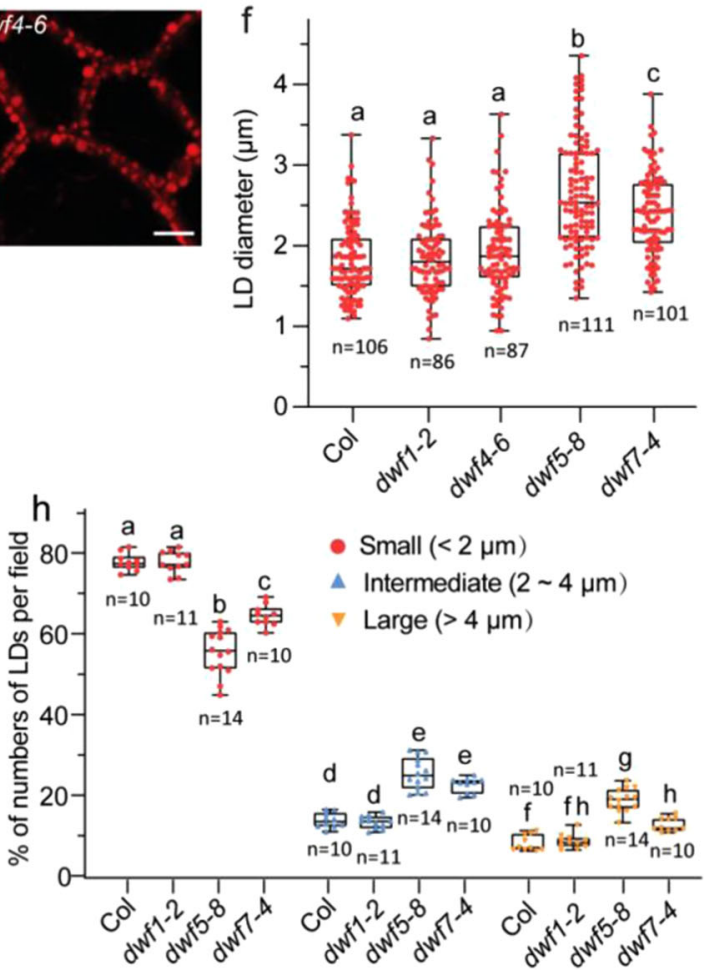

seeds, with increased length of short axis but the comparable length of long axis (Fig. 6a-d).

To examine changes in LD morphology in vivo, 3-day-old seedlings were stained with Nile Red and observed under confocal microscopy. Again, we found that LD size was significantly enlarged in $d w f 5-8, d w f 7-4$ hypocotyl cells than that of WT (Fig. 6e, f). To obtain more quantitative information on changes in LD size, LDs from mature seeds were isolated by floating centrifugation, and numbers of purified LDs were counted and grouped into three different sizes (Fig. 6g, h). The results revealed significant decreases in the number of small LDs in $d w f 5-8$ and $d w f 7-4$ mutants and concomitant increases in the number of intermediate- and large-sized LDs. In addition, there was a significant increase in the number of large-sized LDs at the expense of small-sized ones in $d w f 5-8$ compared with $d w f 7-4$, mirroring more severer decreases in amounts of 24-ethyl- $\Delta^{5}$ sterols in the former than in the later (Supplementary Table 3). No significant difference in the numbers of LDs in all three size categories was found between WT and $d w f 1-2$ (Fig. $6 \mathrm{~g}, \mathrm{~h}$ ). In summary, microscopic analysis of LDs in $d w f 5$ and $d w f 7$ seeds 
Fig. 6 Changes in LD morphology in $\mathbf{d w f 5}$ and $\mathbf{d w f 7}$ mutants. a TEM images of LDs in mature seeds of wild type and $d w f$ mutants. The experiment was repeated 3 times with similar results. Bar $=2 \mu \mathrm{m}$. b Size of LDs. c Long axis to short axis ratios of LDs. d LD number per field of TEM. e Representative confocal images of LD in the hypocotyl epidermis of wild type and dwf mutants. LDs of 3-day-old seedlings were stained with Nile Red and visualized in the hypocotyl epidermis next to the radicle by confocal microscope. The experiment was repeated three times with similar results. Bar $=10 \mu \mathrm{m}$. $\mathbf{f}$ Diameter distribution of LDs from hypocotyl epidermis of wild type and dwf mutants. $\mathbf{g}$ Representative images of isolated LDs. LDs were isolated from dry seeds, stained with Nile Red and visualized with a Zeiss epifluorescence microscope. The experiment was repeated 3 times with similar results. Bar $=20 \mu \mathrm{m}$. h Percentages of different sizes of isolated LDs per microscopic field. Small LDs, diameters $<2 \mu \mathrm{m}$; intermediate LDs, diameters between 2 and $4 \mu \mathrm{m}$; large LDs, diameters larger than $4 \mu \mathrm{m}$. Numbers in $\mathbf{d}$ and $\mathbf{h}$ represent the number of microscopic fields analyzed for data collection. Numbers in $\mathbf{b}, \mathbf{c}$ and $\mathbf{f}$ indicate the number of LDs analyzed. In $\mathbf{b}, \mathbf{c}, \mathbf{d}, \mathbf{f}$ and $\mathbf{h}$, the box plot boundaries reflect the interquartile range, the center line is the median and the whiskers represent $1.5 \times$ the interquartile range from the lower and upper quartiles. Different letters indicate significant differences at $P<0.05$, as determined by one-way ANOVA with Tukey's multiple comparisons test.

reinforces the notion that sterols are required for proper $\mathrm{LD}$ formation.

Seed oleosin protein abundance is markedly decreased in $d w f 5$ and $d w f 7$ mutants. The increased LD size in seeds of $d w f 5$ and $d w f 7$ mutants prompted us to investigate the impact of sterol deficiency on seed oleosin protein levels, since LD size in seeds is known to be determined largely by oleosin protein abundance ${ }^{26}$. For this purpose, we first examined OLE1-GFP signals in developing embryos of sterol mutants overexpressing OLE1. Although there is evidence that the $35 \mathrm{~S} \mathrm{CaMV}$ promoter is not very active in developing seeds ${ }^{34}$, abundant OLE1-GFP-coated LDs were observed in developing embryos of Col/OLE1 and dwf1-2/OLE1 (Fig. 7a). Disruption of DWF5 or DWF7 led to marked decreases in OLE1-GFP-coated LDs in developing embryos of the OLE1 line, and again $d w f 5-8 / O L E 1$ showed a more severe phenotype than $d w f 7-4 / O L E 1$. Western blot analysis confirmed drastic deceases in OLE1-GFP levels in developing embryos of $d w f 5-8 /$ $O L E 1$ and, to a lesser extent, in embryos of $d w f 7-4 / O L E 1$, compared with the Col/OLE1 and $d w f 1-2 / O L E 1$ (Fig. 7b, c). Similarly, the abundance of OLE1-GFP-coated LDs was also found to markedly decrease in cotyledon and root of 6-day-old seedlings of $d w f 5-8 / O L E 1$ and $d w f 7-4 / O L E 1$ compared with Col/OLE1 and dwf1-2/OLE1 (Supplementary Fig. 12).

Oleosins account for up to $79 \%$ of total $\mathrm{LD}$ proteins in seeds of Arabidopsis ${ }^{35}$ and all five oleosin isoforms can be easily resolved by sodium dodecyl sulfate-polyacrylamide gel electrophoresis (SDS-PAGE) $)^{27}$. To gain more quantitative information on the effect of sterol deficiency on oleosin levels, LDs were purified from mature seeds of WT, $d w f 1-2, d w f 5-8$, and $d w f 7-4$, and LDassociated proteins were loaded on a same amount of TAG basis and separated by SDS-PAGE. As expected, the isolated LD fractions were particularly enriched with oleosin proteins migrating within the $10-20 \mathrm{kDa}$ size range (Fig. $7 \mathrm{~d}$ ). Among them, OLE1 was the most abundant isoform, followed by OLE2, OLE3, and OLE4, whereas OLE5, which has the smallest molecular mass ${ }^{27}$, was not detected in our experiments. Compared with WT, both the $d w f 5-8$ and $d w f 7-4$ showed marked decreases in levels of all four oleosin proteins. Results of image analysis showed that the total amounts of OLE1, OLE2, and OLE3 decreased by 50 and $35 \%$ in $d w f 5-8$ and $d w f 7-4$, respectively, compared with the WT, whereas no differences were found between $d w f 1-2$ and WT (Fig. 7e-g). On the other hand, the level of a protein band migrating at $30 \mathrm{kDa}$ (mostly likely the $\mathrm{LD}$ protein caleosin based on its molecular weight) remained largely unaltered in $d w f 5-8$ and $d w f 7-4$ mutants compared with the WT (Fig. 7h).

\section{Discussion}

The lipid and protein factors involved in regulating LD biogenesis and growth remain unknown, particularly in plants. In this study, a comprehensive genetic, biochemical, and cell biological analysis uncovers a BR-independent role of sterols in LD accumulation in plants. Sterols can function as signaling molecules in regulating gene transcription in plants 6 . Our analysis, however, revealed a strong positive correlation between the OLE1 transcript level and LD abundance in $d w f$ mutants and other LD-deficient mutants with normal growth phenotypes. Similarly, TAG and LD accumulation has often been found to concur with increases in oleosin protein and transcript levels in developing seeds. Disruption of WRINKLED1, a key transcription factor controlling the expression of genes involved in the synthesis of fatty acids but not oleosins, results in drastic decreases in levels of both the TAGs and oleosin transcripts in seeds $^{36}$. In addition, TAG content and oleosin expression have been found to decrease concomitantly in seeds of a mutant disrupted in TAG synthesis ${ }^{37}$. Together, these results strongly suggest that oleosin transcript levels are controlled by LD abundance and imply that sterols impact oleosin gene expression indirectly via their effects on LD assembly. Sterols may be involved in the regulation of RNA processing, stability, and translation, but to the best of our knowledge, no such regulatory roles have been reported in any model systems. On the other hand, sterols as key regulators of the physical properties and microdomain organization of cellular membranes are well documented. Therefore, A more plausible explanation for the phenotype is that sterol deficiency or imbalanced sterol composition disrupt normal membrane properties and domain organization necessary for membrane-associated events during the coordinated assembly of LDs from oil and oleosins.

Oleosins function not only as the key determinants of LD stability and size but also in the early stages of LD formation such as LD budding from the ER into cytosol ${ }^{24}$. Arabidopsis seed LDs contain five oleosin proteins, with OLE1, OLE2, and OLE3 as the major isoforms (Fig. 7d). A previous study showed that combined knockout of OLE1, OLE2, and OLE4 genes only results in an about $10 \%$ decrease in seed oil 27 . In $d w f 5-8$ mutant seeds, a $50 \%$ decrease in amounts of OLE1, OLE2, and OLE3 was accompanied by up to $40 \%$ decreases in oil content in mature seeds (Fig. 5e and Supplementary Fig. 10). These results suggest that the decreased oil content in $d w f 5$ seeds cannot be solely explained by changes in oleosin levels. Based on LD size distribution shown in Fig. 6h, we estimated that the increased LD size in $d w f 5$ only resulted in a small decrease (less than 5\% compared with WT) in LD surface area, suggesting that the decreased oleosin levels are not due to a decrease in LD surface area.

Due to their long hydrophobic stretch, oleosins are predicted to be unstable when present in ER membranes and become stable when associated with $\mathrm{LDs}^{25}$. Likely for this reason, the transcript levels of genes involved in oil and oleosin synthesis are coordinately regulated during seed development ${ }^{38}$. In addition, oleosins are synthesized in ER domains alongside with the synthesis of TAGs $^{39}$ and the assembly of nascent $\mathrm{LDs}^{40}$. Presumably, this 

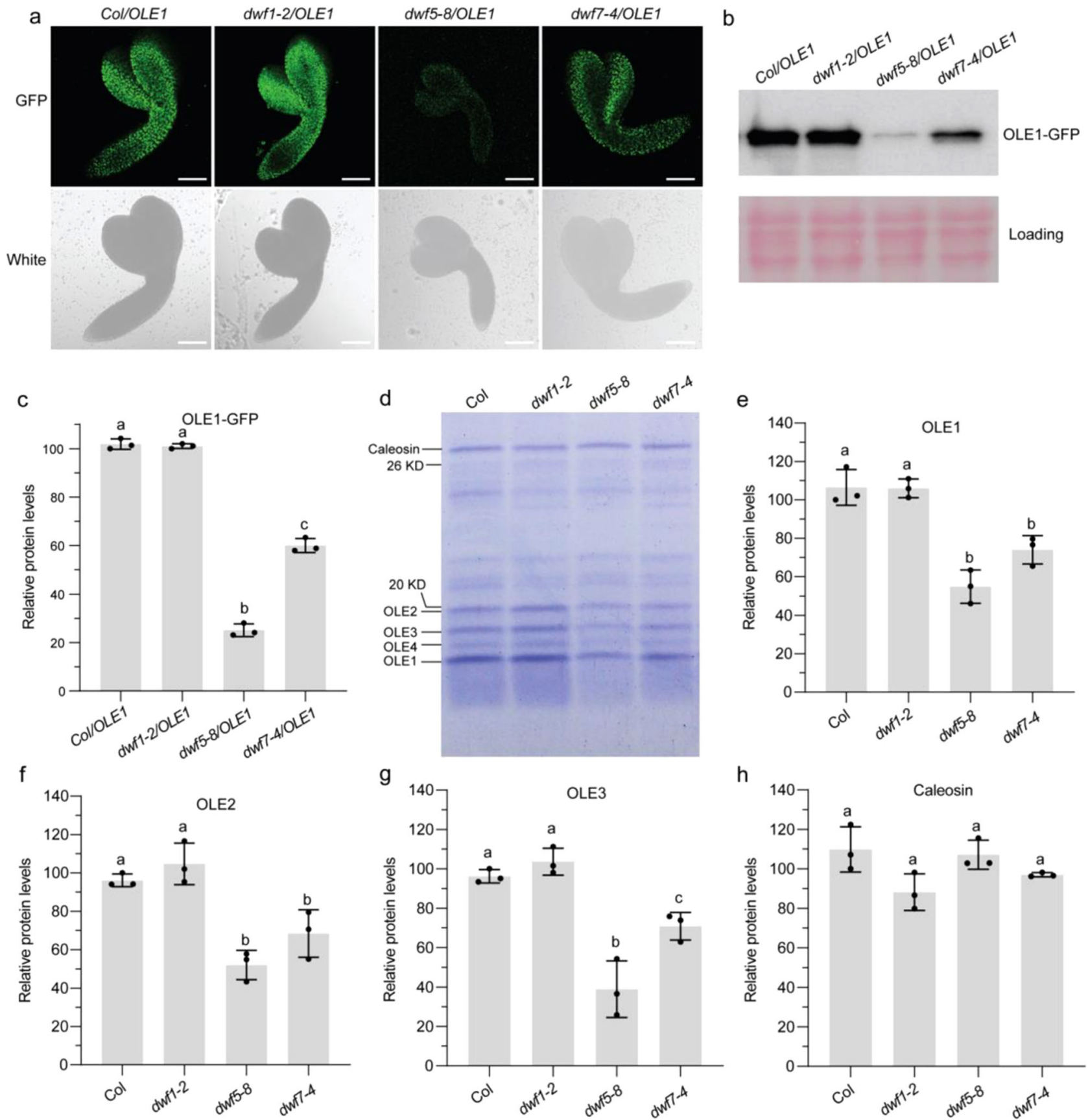

Fig. 7 Seed oleosin protein abundance is decreased in $\mathbf{d w f 5}$ and $\mathbf{d w f 7}$ mutants. a Representative images of LDs (OLE1-GFP, green) in embryos at the bent cotyledon stage. The experiment was repeated three times with similar results. Bar $=100 \mu \mathrm{m}$. b Western blotting with anti-GFP antibody showing OLE1GFP protein levels in embryos of Col/OLE1, dwf1-2/OLE1, dwf5-8/OLE1, and dwf7-4/OLE1 at the bent cotyledon stage. Equal loading of proteins are shown by Ponceau S staining. c Relative OLE1-GFP protein levels quantified using Image J software. $\mathbf{d}$-h, Oleosin and caleosin protein levels in seed LDs. LDs were purified from dry seeds. Proteins of LDs equivalent to $15 \mu \mathrm{g}$ of TAGs were subjected to SDS-PAGE with Coomassie blue staining (d). Relative oleosin and caleosin protein levels were quantified using ImageQuant TL software (e-h). In $\mathbf{c}, \mathbf{e}, \mathbf{f}, \mathbf{g}$, and $\mathbf{h}$, data are mean \pm SD. of three biological replicates. Different letters indicate significant differences at $P<0.05$, as determined by one-way ANOVA with Tukey's multiple comparisons test.

spatiotemporal regulation enables a concomitant accumulation of TAG and oleosins and efficient transfer of oleosins to $\mathrm{LDs}^{25,39,40}$ during seed filling. Analysis of temporal changes in oil accumulation in developing seeds showed that the decrease in TAG content was much more pronounced in the bent cotyledon than in the green mature stage of developing embryos in both the $d w f 5$ and $d w f 7$ mutants (Supplementary Fig. 11). During early embryo development, $d w f 5$ and $d w f 7$ mutants contained $57-73 \%$ lower levels of TAG than $d w f 1$. If oleosin synthesis in $d w f 5$ and $d w f 7$ mutants continued at a similar rate to $d w f 1$, most of these nascent proteins would be expected to remain in the ER and be degraded quickly. During the transition from the bent cotyledon to the green mature stage, the rates of TAG accumulation were threefold higher in $d w f 5$ and $d w f 7$ than the rate found in $d w f 1$. Such high rates of TAG accumulation may not be matched by a similar rate of oleosin synthesis. Together, these results suggest that disruption of the coordinated synthesis of TAG and oleosins and their deposition into LDs may be one of the major factors contributing 
to the formation of LDs with the less oleosin coat in mutants deficient in sterols.

Given so much is still unknown regarding the mechanism of LD biogenesis, we can only speculate on the functions of sterols in neutral lipid storage in plants. Like DAG, sterols are non-bilayer, conical lipids with a small polar head group and a large hydrophobic body. When present in the biological membranes, sterols induce lipid packing defects, increase membrane surface tension and alter other aspects of membrane properties in a similar way as DAG does ${ }^{41,42}$. In addition, the presence of sterols has been shown to greatly amplify the membrane perturbing effects of $\mathrm{DAG}^{43}$. DAG has been suggested as one of the key lipids in regulating membrane biophysical properties and hence initial oil lens formation as well as LD budding ${ }^{18,44,45}$. Therefore, it is possible that sterols, either acting alone or synergistically with DAG, are involved in regulating membrane properties necessary for LD formation. Indeed, a recent study has shown that cholesterol promotes LD initiation and the packaging of TAGs into $\mathrm{LDs}^{45}$. In addition to its roles in the early steps of $\mathrm{LD}$ biogenesis, DAG has been shown to be important for protein targeting to $\mathrm{LDs}^{46}$, likely via its ability to induce local phospholipid packing defects on LD surface which function as membrane docking sites for some of LD-associated proteins ${ }^{44}$. Free sterols are known to be amply present in the LD phospholipid monolayer ${ }^{47,48}$. Therefore, the second possible scenario is that deficiency in sterols impairs the ability of LD monolayer surface lipids to induce lipid packing defects and hence the recruitment of LDassociated proteins necessary for $\mathrm{LD}$ initiation, budding, growth, and protection. In line with this, sterols have been shown to affect the physical properties of the LD surface and hence the targeting of TAG-synthesizing enzymes to LDs in mammals ${ }^{49}$. The third possibility is that deficiency in sterols impairs the ability of ER membranes to generate sterol-rich microdomains necessary for LD biogenesis. In support, there is ample evidence that LDs are formed at discrete sites of the $\mathrm{ER}^{17,18,44}$. In addition, sterol-rich ER domains have been implicated in LD biogenesis ${ }^{10,11}$ and the synthesis of neutral and membrane lipids ${ }^{9}$. In mammalian cells, disruption of lipid microdomains enriched in cholesterol inhibits LD formation ${ }^{11}$.

The most important structural determinants of sterols in modulating membrane properties and domain organization are the presence of a side chain of 8 to 10 carbon length at position C-17, a hydroxyl group at position C-3, and a $\Delta^{5}$ double bond between carbon atoms C-5 and C-6 in the tetracyclic ring ${ }^{50-52}$. In particular, it has been shown that the $\Delta^{5}$ double bond is required for the preferential interaction of sterols with saturated phospholipids and this interaction serves as a driving force for the formation of membrane microdomains ${ }^{53-55}$. Importantly, a shift of the double bond position or introduction of a second double bond in the tetracyclic ring changes the membrane domain forming properties of the sterols ${ }^{56,57}$ and renders sterols far less effective in mediating the sterol-phospholipid interaction ${ }^{50}$. The importance of the $\Delta^{5}$-sterols in regulating membrane properties and hence LD biogenesis is clearly illustrated in $d w f 5$ and $d w f 7$ mutants, which accumulate unusual sterols at the expense of $\Delta^{5}$. sterols and are severely defective in LD formation. On the other hand, disruption of DWF1 causes drastic decreases in sitosterol and campesterol with concomitant increases in isofucosterol and 24-methylenecholesterol but no significant effects on total $\Delta^{5}$-sterol content and LD formation. These results suggest that isofucosterol and 24-methylenecholesterol are functionally interchangeable with sitosterol and campesterol, respectively in LD biogenesis in plants. In fact, isofucosterol and 24methylenecholesterol differ structurally from sitosterol and campesterol only by a single double bond at C-24 of the side chains and the key structural features required for optimal interaction with phospholipids are present in both types of sterols.

Among the major end products of plant sterols, campesterol exhibits the strongest ability to promote membrane domain formation and to organize sterol spatial distribution in membranes ${ }^{57}$. Therefore, a drastic increase in campesterol with a concomitant decrease in sitosterol may disrupt the balanced ratio of campesterol to sitosterol thus the optimal membrane environments necessary for LD formation and thus contribute to $\mathrm{LD}$ deficiency phenotype in cvp1-3 smt3-1. In addition to disturbed sterol composition, changes in overall sterol content appear also to impact LD biogenesis in plants. This notion is supported by several lines of evidence. First, total free sterol levels are reduced only in $d w f 5$ mutants severely deficient in LD biogenies. Second, among all the sterol mutants analyzed, $d w f 5$ has the lowest level of free sterols and this mutant exhibits the most severe LD deficiency phenotype in both leaves and seeds. Third, leaf senescence is known to be associated with an increased accumulation of sterols ${ }^{13}$, TAG levels, LD number (Figs. 1-3) and total sterol content (Supplementary Fig. 13) are all increased in old leaves of both $d w f 5$ and $d w f 7$. Fourth, $d w f 5$ and $d w f 7$ mutants contained higher levels of 24-ethyl- $\Delta^{5}$-sterols in seeds (Supplementary Table 3) than in leaves (Supplementary Table 1) and both mutants showed less severe defects in storage lipid accumulation in seeds than in leaves. Since most of the seed mass is storage lipids and proteins, it seems likely that actual levels of free sterols in cellular membranes of $d w f 5$ and $d w f 7$ mutants are much higher than those reported in Supplementary Table 3. Alternatively, the observed more pronounced defects in LD accumulation in leaves than in seeds may reflect a different mechanistic basis underlying LD biogenesis between these two organs.

In summary, this study reveals a previously unrecognized role of sterols in LD biogenesis in plants. We propose that sterols regulate $\mathrm{LD}$ formation by controlling physicochemical properties of membranes or ER membrane domain organization. Further studies are required to dissect the exact mechanism by which changes in sterol composition and content affect LD formation in plants.

\section{Methods}

Plant materials and growth conditions. Arabidopsis thaliana Columbia- 2 ecotype was used for all experiments described. The mutants $d w f 1-2(\mathrm{CS} 8100)^{58}, d w f 5-8$ $(\text { SALK_127066) })^{33}$, bri1-3 ${ }^{59}$, cvp1-3smt3- ${ }^{60}$, pdat1-2 ${ }^{31}$, psat1-1 (SALK_037289), and psat1-2 (SALK_117091) ${ }^{13}$ were described previously. The dwf4-6 (CS449028) and $d w f 7-4$ (CS442813) mutants were obtained from the Arabidopsis Biological Resource Centre (ABRC) and verified by PCR. The OLE1-GFP overexpressing line was described in Fan et al. ${ }^{31}$.

Surface-sterilized seeds were imbibed for 2 days at $4{ }^{\circ} \mathrm{C}$ in dark and then germinated on half-strength Murashige and Skoog (MS) medium with $0.6 \%(\mathrm{w} / \mathrm{v})$ agar and $1 \%(\mathrm{w} / \mathrm{v})$ sucrose in an incubator $\left(22 / 18^{\circ} \mathrm{C}\right.$ day/night temperature; $16-\mathrm{h}$ light/8-h-dark photoperiod; and $80-120 \mu \mathrm{mol} \mathrm{m}^{-2} \mathrm{~s}^{-1}$ light intensity). Ten-day-old seedlings were transferred to soil and grown in a climate-controlled growth chamber $\left(22 / 18^{\circ} \mathrm{C}\right.$ day/night temperature; 16 -h-light/8-h-dark photoperiod; and $100-150 \mu \mathrm{mol} \mathrm{m}^{-2} \mathrm{~s}^{-1}$ light intensity).

Isolation of M1-7, map-based cloning, and whole-genome sequencing. Seeds of the transgenic plants overexpressing OLE1 fused with GFP were mutagenized with ethyl methanesulfonate (EMS) and leaves of 3-week-old $\mathrm{M}_{2}$ plants were used to screen LD mutants using an epifluorescence microscope (Carl Zeiss; Axiovert $200 \mathrm{M}$ ). One dwarf mutant named M1-7 with only very few LDs was isolated. For map-based cloning, the M1-7 mutant was crossed with the wildtype plants of Landsberg erecta ecotype. About 60 F2 plants with M1-7 mutant phenotype were used for rough mapping. Mapping markers (Supplementary Table 4) were designed according to Arabidopsis mapping platform (AMP) website (http://amp.genomics.org.cn//) 61

For the whole-genome sequencing, young leaf tissue from $102 \mathrm{~F}_{2}$ lines of the mapping population were pooled, genomic DNA was extracted using DNeasy plant Mini Kit (QIAGEN, cat\# 69104) and the genomic library preparation and sequencing were done in Weill Cornell Medical College Genomics Resources Center. The DNA was sequenced using a HiSeq2500 (Illumina) with 100-bp singleend reads. The clean reads were aligned to the WT reference genome and reliable 
SNPs in the region of chromosome 1, where the mutation of M1-7 was delimited by rough mapping, were identified.

Lipid and fatty acid analyses. For leaf TAG quantification, neutral lipids were extracted from leaves with chloroform/methanol/formic acid (1:2:0.1, by volume) and separated on silica plates (Silica Gel 60, EMD Millipore Corporation) by thinlayer chromatography (TLC) using a solvent system consisting of hexane/diethyl ether/acetic acid (70:30:1, by volume). TAG on TLC plates was visualized by spraying $5 \% \mathrm{H}_{2} \mathrm{SO}_{4}$ followed by charring and the relative TAG band intensity was measured using the ImageJ software. Seeds harvested from plants grown side-byside in the same flat were used for seed TAG quantification. In brief, 15 dry seeds or geminating seeds were incubated in $1 \mathrm{ml}$ of $2.5 \%$ sulfuric acid in methanol (v/v) at $90^{\circ} \mathrm{C}$ for $2 \mathrm{~h}$. Five microgram C17:0 was added as an internal standard prior to transmethylation. Fatty acyl methyl esters were extracted with hexane and quantified by an HP5975 gas chromatography-mass spectrometer (Hewlett-Packard) fitted with $60 \mathrm{~m} \times 250-\mu \mathrm{m}$ SP-2340 capillary column (Supelco) ${ }^{31}$.

Microscopy. Fresh leaf tissue was mounted on slides with water and observed under a Zeiss epifluorescence microscope (Carl Zeiss; Axiovert $200 \mathrm{M}$ ) equipped with a GFP filter set 38 (Carl Zeiss). For LD imaging in hypocotyl, 3-day-old seedlings germinated on $1 / 2$ MS medium without sucrose were stained with $10 \mu \mathrm{g} /$ $\mathrm{ml}$ Nile Red (Sigma-Aldrich) and examined using a Leica TCS SP5 laser scanning confocal microscope with excitation at $543 \mathrm{~nm}$ and emission at 570-700 nm. For leaf LD imaging in oleic acid feeding experiments, leaves were washed with water for four times with shake ( $5 \mathrm{~min}$ each) after feeding, blotted dry, then stained with $10 \mu \mathrm{g} / \mathrm{ml}$ BODIPY 558/568 C12 (Invitrogen, cat\# D3835) and examined by confocal microscopy. For transmission electron microscopy (TEM), mature green embryos of the same developmental stage at about 13 to14 days after pollination were collected, and the Ultrabed Kit (Electron Microscopy Sciences) were used for infiltration and embedding. Samples were then sectioned and stained with $2 \%$ uranyl acetate and lead citrate before viewing under a JEM-1400 LaB6 120-keV transmission electron microscope (JEOL, USA). The size of LDs was measured using the ImageJ software.

Seed and LD size measurements. Seeds from different genotype were harvested at the same time from plants grown side-by-side in the same flat. Seeds pictures were taken with Leica M125 dissecting microscope with Jenoptik ProgRes SpeedXT Core 5 CCD microscope camera. The length and width of the seeds were measured using the ImageJ software.

Sterol measurements by GC-MS. Free sterols were extracted as described previously ${ }^{62}$ with some modifications. Briefly, about $2 \mathrm{~g}$ mature leaf of 4 -week-old plants or $0.1 \mathrm{~g}$ dry seeds were used for total lipid extraction in $20 \mathrm{ml}$ chloroform/ methanol/formic acid (1/2/0.1, by volume) for $1 \mathrm{~h}$ at room temperature by vigorous shaking. Then $10 \mathrm{ml}$ of $1 \mathrm{M} \mathrm{KCl}-0.2 \mathrm{M} \mathrm{H}_{3} \mathrm{PO}_{4}$ was added. Following centrifugation at $10,000 \mathrm{~g}$ for $5 \mathrm{~min}$, the lower organic fractions were transferred to new tubes, and $10 \mathrm{ml}$ chloroform/methanol (1:2, by volume) was added to the remaining upper phase. After centrifugation, the lower organic phases were pooled and evaporated to dryness under $\mathrm{N}_{2}$. Dried lipids were resuspended in $1 \mathrm{ml}$ hexane and centrifuged for 5 min at 10,000 g. $0.5 \mathrm{ml}$ lipids were spotted on a TLC plate and separated with dichloromethane as a developing solvent. TLC plates were spray-stained with ethanolic Berberine sulfate solution $(0.01 \%, w / v)$ and free sterols were visualized by fluorescence $(A=366 \mathrm{~nm})$. Bands containing free sterol $(\mathrm{RF}=0.2-0.3)$ and sterylester $(\mathrm{Rf}=0.9)$ fractions were scraped off. Free sterols were extracted with $3 \mathrm{ml}$ of chloroform/methanol/formic acid (1/2/0.1, by volume) in the presence of $5 \mu \mathrm{g} 5$ - $\alpha$ Cholestane as an internal standard. Then, $1.5 \mathrm{ml} 1 \mathrm{M} \mathrm{KCl}-0.2 \mathrm{M} \mathrm{H}_{3} \mathrm{PO}_{4}$ was added to the extract and the mixture was vortexed vigorously. After $5 \mathrm{~min}$ of centrifugation at $10,000 \mathrm{~g}$, the organic fractions were transferred to new glass tubes, dried under $\mathrm{N}_{2}$. Steryl-ester fractions were heated for $1 \mathrm{~h}$ at $90{ }^{\circ} \mathrm{C}$ with $6 \%$ potassium hydroxide in ethanol and the liberated free sterols were extracted with hexane. The sterols were then subjected to silylation in $100 \mu \mathrm{l} \mathrm{N}$-Methyl-N-(trimethylsilyl) trifluoroacetamide (MSTFA) for $2 \mathrm{~h}$ at $70^{\circ} \mathrm{C}$. After the reagents were evaporated under $\mathrm{N}_{2}$, the sterol derivatives were dissolved in hexane and used for GC-MS analyses. The analysis of sterol derivatives was performed on an HP5975 gas chromatography-mass spectrometer (Hewlett-Packard) fitted with a J\&W DB$5 \mathrm{~ms}$ capillary column ( $30 \mathrm{~m}$ long, $0.25 \mathrm{~mm}$ inner diameter, film thickness $0.25 \mu \mathrm{m}$ ) with helium as a carrier gas. The temperature program used was $170^{\circ} \mathrm{C}$ for $1.5 \mathrm{~min}$, a fast increase from 170 to $280{ }^{\circ} \mathrm{C}$ and a slow increase from 280 to $300^{\circ} \mathrm{C}$. The inlet temperature was $280^{\circ} \mathrm{C}$. The sterols were identified based on their characteristic retention times and on well-documented fragmentation patterns of their trimethylsilyl derivatives ${ }^{63}$

RNA extraction and quantitative reverse transcription PCR (qRT-PCR). Total RNAs were extracted from mature leaves of 4-week-old plants using the TRIzol reagent (Thermo Fisher Scientific, cat\# 15596026). First-strand cDNA was synthesized using M-MLV reverse transcriptase (New England Biolabs, cat\# M0253). qRT-PCR was performed with SsoAdvanced Universal SYBR Green Supermix (Bio-Rad, cat\# 1725270). The results were normalized to expression levels of UBQ5. The primers used for the qRT-PCR analysis are listed in Supplementary Table 4.
Oleic acid feeding. Oleic acid was dispersed and suspended in an incubation buffer (20 mM MES, pH 5.5, 0.01\% Tween 20, one-tenth strength of MS salts, $0.5 \mathrm{mM}$ oleic acid) by sonication for $15 \mathrm{~s}$. Detached mature leaves of 5-week-old plants were then floated in the incubation buffer in the light $\left(60 \mu \mathrm{mol} \mathrm{m}^{-2} \mathrm{~s}^{-1}\right)$ at room temperature with gentle shaking for $12 \mathrm{~h}$. The leaves were washed with water for four times ( $5 \mathrm{~min}$ each) and incubated in the same medium lacking oleic acid for $24 \mathrm{~h}$. Total lipids were extracted and separated by TLC as described above. The TAG bands were visualized by brief exposure to iodine vapor and TAG was quantified by GC-MS.

In vivo acetate and oleic acid pulse-chase labeling. In vivo ${ }^{14} \mathrm{C}$-acetate pulsechase assay was carried out according to Fan et al. ${ }^{64}$. Briefly, rapidly growing leaves of 5 -week-old plants were detached and labeled for $1 \mathrm{~h}$ with ${ }^{14} \mathrm{C}$-acetate. After washing three times with water, the leaves were incubated further with unlabeled solution (20 mM MES, pH 5.5, one-tenth strength of MS salts, and $0.01 \%(\mathrm{v} / \mathrm{v})$ Tween 20) under a 16 -h-light/8-h-dark cycle for $16 \mathrm{~h}$ to $36 \mathrm{~h}$. Total lipids were extracted and separated by TLC as described above. Label incorporation into tota lipids was determined by liquid scintillation counting. The ${ }^{14} \mathrm{C}$-labeled TAG was separated by TLC and analyzed by phosphorimaging using a Typhoon FLA 7000 imager (GE Healthcare). Relative radioactivity of the TAG bands was quantified using the ImageQuant5.2 software.

For ${ }^{14} \mathrm{C}$-oleic acid pulse-chase labeling assay, mature leaves of 5 -week-old plants were incubated with $0.02 \mathrm{mM}{ }^{14} \mathrm{C}$-oleic acid $(50 \mathrm{mCi} / \mathrm{mM}$, American Radiolabeled Chemicals) and then chase for $24 \mathrm{~h}$ as described above. Total lipids were extracted and separated by TLC. The radiolabeled TAG bands were visualized by brief exposure to iodine vapor and the radioactivity in TAG was quantified by liquid scintillation counting.

LD isolation, LD size measurements, and LD protein analysis. Seed LDs were isolated from Arabidopsis seed following the protocol from Ding et al. ${ }^{65}$ with some modifications. Briefly, $40 \mathrm{mg}$ dry seeds were ground in $5 \mathrm{~mL}$ of ice-cold buffer A (20 mM tricine, pH 7.8, $250 \mathrm{mM}$ sucrose, $10 \mathrm{mM} \mathrm{KCl,} 1 \mathrm{mM}$ EDTA, $1 \mathrm{mM} \mathrm{MgCl}$, $5 \mathrm{mM} \beta$-mercaptoethanol, and $1 \mathrm{mM}$ PMSF, $1 \times$ cocktail) using a mortar and pestle. The crude homogenate was centrifuged at $3000 \times \mathrm{g}$ for $10 \mathrm{~min}$ and the supernatant (named postnuclear supernatant, PNS) was transferred to a $15 \mathrm{ml}$ glass tube. Then $8 \mathrm{ml}$ of buffer B (20 mM Hepes, pH 7.4, $100 \mathrm{mM} \mathrm{KCl}, 1 \mathrm{mM}$ EDTA, $2 \mathrm{mM} \mathrm{MgCl}_{2}, 5 \mathrm{mM} \beta$-mercaptoethanol, and $1 \mathrm{mM} \mathrm{PMSF}, 1 \times$ cocktail) was loaded on top of the PNS. Following centrifugation at 10,200 g using HB-6 SwingingBucket Rotor for $1 \mathrm{~h}$ at $4{ }^{\circ} \mathrm{C}$, the LDs from the top LD fraction were carefully collected, suspended them in $1 \mathrm{ml}$ buffer B, and then centrifuged at $17,000 \mathrm{~g}$ for $5 \mathrm{~min}$ at $4{ }^{\circ} \mathrm{C}$. The LD fraction was suspended in $200 \mu \mathrm{l}$ buffer B and used for further analysis.

To examine LD morphology, LDs were stained with Nile red, and viewed using a Zeiss epifluorescence microscope (Carl Zeiss; Axiovert $200 \mathrm{M}$ ) with a red fluorescent protein filter. The size and number of LDs were quantified using ImageJ software.

For TAG quantification and protein analysis of LDs, $1 \mu \mathrm{l}$ of well suspended LDs were added to $60 \mu \mathrm{l}$ chloroform to extract the lipids and the lipids were separated by TLC. Relative contents of TAG in $1 \mu \mathrm{l}$ LDs from different genotypes were quantified using ImageJ software. LDs containing a similar amount of TAG were delipidated in $0.5 \mathrm{ml}$ chloroform/acetone $(1: 1, \mathrm{v} / \mathrm{v})$. After vortex and centrifugation $(10000 \mathrm{~g} \times 5 \mathrm{~min}), 400 \mu \mathrm{l}$ lipids in the organic phase were transferred to a new tube and TAG concentration was measured by GC-MS. The leftover lipids, upper phase and pellet were air-dried and dissolved in $2 \times$ laemmli sample buffer. Proteins from LDs containing $15 \mu \mathrm{g}$ of TAG were separated by $15 \%$ SDS-PAGE gel, followed by staining with Coomassie blue. The gel was visualized using ImageQuant LAS 4000 biomolecular imager (GE Healthcare Life Sciences), and relative protein levels were quantified using ImageQuant TL software according to the manual.

PDAT1 activity assay. Microsomal membranes were prepared according to Xu et al. ${ }^{66}$. In general, about $1.5 \mathrm{~g}$ mature leaves from 5 -week-old plants were powdered with liquid nitrogen in a mortar and pestle. A grinding medium (100 mM HEPES $\mathrm{KOH}, \mathrm{pH} 7.4$, containing $1 \mathrm{mM}$ EDTA, $0.32 \mathrm{M}$ sucrose, and $1 \mathrm{mM}$ dithiothreitol) was then added. The homogenates were filtered through two layers of Miracloth (Calbiochem, Gibbstown, NJ, USA), and centrifuged at $3000 \mathrm{~g}$ for $5 \mathrm{~min}$, and the supernatant re-centrifuged at $15,000 \mathrm{~g}$ for $15 \mathrm{~min}$. The supernatant was recentrifuged at $120,000 \mathrm{~g}$ for $1 \mathrm{~h}$ and the resulting pellet was resuspended in grinding medium, sonicated for $30 \mathrm{~s}$ and protein concentrations were determined using Pierce BCA Protein Assay Kit (Thermo-Scientific, cat\#23227). To prepare ${ }^{14} \mathrm{C}$-labeled $\mathrm{PC}^{64}$, two-week-old seedlings were incubated in $20 \mathrm{mM} \mathrm{MES}-\mathrm{KOH}$, pH 6.0, with $0.2 \mathrm{mCi}$ of ${ }^{14} \mathrm{C}$-acetate (American Radiolabeled Chemicals) for overnight. Total lipids were extracted and separated by TLC. ${ }^{14} \mathrm{C}$-labeled PC was eluted from silica gel using chloroform: methanol: formic acid (1:2:0.1, by volume) and redissolved in chloroform.

PDAT1 activity assay was conducted in a reaction mixture $(100 \mu \mathrm{l})$ containing $100 \mathrm{mM}$ HEPES-KOH (pH 7.4), $250 \mu \mathrm{M}{ }^{14} \mathrm{C}$-labeled PC and $250 \mu \mathrm{M}$ 18:1-DAG (Avanti Polar Lipids), 0.5 mM ATP, $0.5 \mathrm{mM}$ Coenzyme A, $1 \mathrm{mM} \mathrm{MgCl}_{2}, 0.02 \%$ Tween 20 . The reaction mixture was thoroughly mixed and incubated at $30^{\circ} \mathrm{C}$ for 
$30 \mathrm{~min}$. The ${ }^{14} \mathrm{C}$-labelled TAGs were isolated by TLC and the radioactivity was determined by scintillation counting.

Immunoblotting. Embryos were dissected under dissecting microscope. Fifty embryos at the bent cotyledon stage were grounded with liquid $\mathrm{N}$ in the protein extraction buffer (50 mM HEPES-KOH, pH 7.6, $150 \mathrm{mM} \mathrm{NaCl}, 1 \mathrm{mM}$ EDTA, 10\% glycerol, $1 \mathrm{mM} \beta$-mercaptoethanol, $0.25 \%$ Triton-X 100, and $1 \times$ complete protease inhibitor cocktail). Protein content was quantified using pierce BCA protein assay kit (ThermoFisher, cat\#23227) according to the manufacturer's instructions. Proteins were separated by $12 \%$ SDS-PAGE gel and blotted to a PVDF membrane. OLE1-GFP protein was detected using mouse anti-GFP antibody (1:3000, Clontech, cat\# 632380), and then with goat antimouse IgG HRP-conjugated secondary antibodies. HRP activity was detected using SuperSignal western detection reagents (Thermo Fisher Scientific) and visualized with an ImageQuant LAS 4000 biomolecular imager (GE Healthcare Life Sciences). The band intensity was measured using ImageJ software.

Reporting Summary. Further information on research design is available in the Nature Research Reporting Summary linked to this article.

\section{Data availability}

Data supporting the finding of this work are available within the paper and its supplementary information files. Raw GC-MS data generated for quantification of TAGs and sterols are available upon reasonable request. Whole-genome sequencing data used during positional cloning are deposited in NCBI's SRA (Sequence Read Archive) under the accession number PRJNA760508. Source data are provided with this paper.

Received: 8 March 2021; Accepted: 9 September 2021;

Published online: 22 September 2021

\section{References}

1. Boutte, Y. \& Grebe, M. Cellular processes relying on sterol function in plants. Curr. Opin. Plant Biol. 12, 705-713 (2009).

2. Valitova, J. N., Sulkarnayeva, A. G. \& Minibayeva, F. V. Plant sterols: Diversity, biosynthesis, and physiological functions. Biochem. (Mosc.) 81, 819-834 (2016).

3. Hartmann, M. A. Plant sterols and the membrane environment. Trends Plant Sci. 3, 170-175 (1998).

4. Planas-Riverola, A. et al. Brassinosteroid signaling in plant development and adaptation to stress. Development 146, dev151894 (2019).

5. Lung, S. C. et al. Arabidopsis ACYL-COA-BINDING PROTEIN1 interacts with STEROL C4-METHYL OXIDASE1-2 to modulate gene expression of homeodomain-leucine zipper IV transcription factors. N. Phytol. 218, 183-200 (2018).

6. He, J. X. et al. Sterols regulate development and gene expression in Arabidopsis. Plant Physiol. 131, 1258-1269 (2003).

7. Malinsky, J., Opekarova, M., Grossmann, G. \& Tanner, W. Membrane microdomains, rafts, and detergent-resistant membranes in plants and fungi. Annu. Rev. Plant Biol. 64, 501-529 (2013).

8. Keinath, N. F. et al. PAMP (Pathogen-associated Molecular Pattern)-induced changes in plasma membrane compartmentalization reveal novel components of plant immunity. J. Biol. Chem. 285, 39140-39149 (2010).

9. Area-Gomez, E. et al. Upregulated function of mitochondria-associated ER membranes in Alzheimer disease. EMBO J. 31, 4106-4123 (2012).

10. Kassan, A. et al. Acyl-CoA synthetase 3 promotes lipid droplet biogenesis in ER microdomains. J. Cell Biol. 203, 985-1001 (2013).

11. Kimura, H., Arasaki, K., Iitsuka, M. \& Tagaya, M. Syntaxin 17 recruits ACSL3 to lipid microdomains in lipid droplet biogenesis. Contact 2, 251525641983871 (2019).

12. Benveniste, P. Biosynthesis and accumulation of sterols. Annu. Rev. Plant Biol. 55, 429-457 (2004)

13. Bouvier-Nave, P. et al. Involvement of the phospholipid sterol acyltransferase 1 in plant sterol homeostasis and leaf senescence. Plant Physiol. 152, 107-119 (2010).

14. Chapman, K. D., Aziz, M., Dyer, J. M. \& Mullen, R. T. Mechanisms of lipid droplet biogenesis. Biochem. J. 476, 1929-1942 (2019).

15. Thiam, A. R. \& Ikonen, E. Lipid droplet nucleation. Trends Cell Biol. 31, 108-118 (2021).

16. Choudhary, V. \& Schneiter, R. Lipid droplet biogenesis from specialized ER subdomains. Microb. Cell 7, 218-221 (2020).

17. Nettebrock, N. T. \& Bohnert, M. Born this way - Biogenesis of lipid droplets from specialized ER subdomains. Biochim. Biophys. Acta Mol. Cell Biol. Lipids 1865, 158448 (2020).
18. Gao, M., Huang, X., Song, B. L. \& Yang, H. The biogenesis of lipid droplets: lipids take center stage. Prog. Lipid Res. 75, 100989 (2019)

19. Choudhary, V. et al. Architecture of lipid droplets in endoplasmic reticulum is determined by phospholipid intrinsic curvature. Curr. Biol. 28, 915-926 (2018). e919.

20. Santinho, A. et al. Membrane curvature catalyzes lipid droplet assembly. Curr Biol. 30, 2441-2894 (2020).

21. Ben M'barek, K. et al. ER membrane phospholipids and surface tension control cellular lipid droplet formation. Dev. Cell 41, 591-604 (2017). e597.

22. Thiam, A. R. \& Beller, M. The why, when and how of lipid droplet diversity. J. Cell Sci. 130, 315-324 (2017).

23. Deslandes, F., Thiam, A. R. \& Foret, L. Lipid droplets can spontaneously bud off from a symmetric bilayer. Biophys. J. 113, 15-18 (2017)

24. Huang, C. Y. \& Huang, A. H. C. Unique motifs and length of hairpin in oleosin target the cytosolic side of endoplasmic reticulum and budding lipid droplet. Plant Physiol. 174, 2248-2260 (2017).

25. Hsieh, K. \& Huang, A. H. C. Endoplasmic reticulum, oleosins, and oils in seeds and tapetum cells. Plant Physiol. 136, 3427-3434 (2004).

26. Siloto, R. M. P. et al. The accumulation of oleosins determines the size of seed oilbodies in Arabidopsis. Plant Cell 18, 1961-1974 (2006)

27. Miquel, M. et al. Specialization of oleosins in oil body dynamics during seed development in Arabidopsis seeds. Plant Physiol. 164, 1866-1878 (2014)

28. Eberle, D., Hegarty, B., Bossard, P., Ferre, P. \& Foufelle, F. SREBP transcription factors: master regulators of lipid homeostasis. Biochimie $\mathbf{8 6}$ 839-848 (2004)

29. Harker, M. et al. Co-ordinate regulation of sterol biosynthesis enzyme activity during accumulation of sterols in developing rape and tobacco seed. Planta 216, 707-715 (2003).

30. Katayama, M. \& Katoh, M. Soybean sterols during maturation of seeds .1. accumulation of sterols in free form, fatty-acid esters, acylated glucosides and non-acylated glucosides. Plant Cell Physiol. 14, 681-688 (1973).

31. Fan, J., Yan, C., Zhang, X. \& Xu, C. Dual role for phospholipid:diacylglycerol acyltransferase: enhancing fatty acid synthesis and diverting fatty acids from membrane lipids to triacylglycerol in Arabidopsis leaves. Plant Cell $\mathbf{2 5}$ 3506-3518 (2013).

32. Clouse, S. D. Arabidopsis mutants reveal multiple roles for sterols in plant development. Plant Cell 14, 1995-2000 (2002).

33. Choe, $\mathrm{S}$. et al. Lesions in the sterol delta reductase gene of Arabidopsis cause dwarfism due to a block in brassinosteroid biosynthesis. Plant J. 21, 431-443 (2000).

34. Williamson, J. D., Hirschwyncott, M. E., Larkins, B. A. \& Gelvin, S. B. Differential accumulation of a transcript driven by the camv s-35 promoter in transgenic tobacco. Plant Physiol. 90, 1570-1576 (1989).

35. Jolivet, P. et al. Protein composition of oil bodies in Arabidopsis thaliana ecotype WS. Plant Physiol. Biochem. 42, 501-509 (2004).

36. Maeo, K. et al. An AP2-type transcription factor, WRINKLED1, of Arabidopsis thaliana binds to the AW-box sequence conserved among proximal upstream regions of genes involved in fatty acid synthesis. Plant $J$. 60, 476-487 (2009).

37. Zou, J., Brokx, S. J. \& Taylor, D. C. Cloning of a cDNA encoding the $21.2 \mathrm{kDa}$ oleosin isoform from Arabidopsis thaliana and a study of its expression in a mutant defective in diacylglycerol acyltransferase activity. Plant Mol. Biol. 31 429-433 (1996).

38. Ruuska, S. A., Girke, T., Benning, C. \& Ohlrogge, J. B. Contrapuntal networks of gene expression during Arabidopsis seed filling. Plant Cell 14, 1191-1206 (2002).

39. Lacey, D. J., Beaudoin, F., Dempsey, C. E., Shewry, P. R. \& Napier, J. A. The accumulation of triacylglycerols within the endoplasmic reticulum of developing seeds of Helianthus annuus. Plant J. 17, 397-405 (1999).

40. Sarmiento, C., Ross, J. H. E. \& Murphy, D. J. Expression and subcellular targeting of a soybean oleosin in transgenic rapeseed. Implications for the mechanism of oil-body formation in seeds. Plant J. 11, 783-796 (1997).

41. Subczynski, W. K., Pasenkiewicz-Gierula, M., Widomska, J., Mainali, L. \& Raguz, M. High cholesterol/low cholesterol: effects in biological membranes. Cell Biochem. Biophys. 75, 369-385 (2017).

42. Alwarawrah, M., Dai, J. \& Huang, J. Modification of lipid bilayer structure by diacylglycerol: a comparative study of diacylglycerol and cholesterol. J. Chem. Theory Comput. 8, 749-758 (2012).

43. Coorssen, J. R. \& Rand, R. P. Effects of cholesterol on the structural transitions induced by diacylglycerol in phosphatidylcholine and phosphatidylethanolamine bilayer systems. Biochem. Cell Biol. 68, 65-69 (1990).

44. Olzmann, J. A. \& Carvalho, P. Dynamics and functions of lipid droplets. Nat. Rev. Mol. Cell Biol. 20, 137-155 (2019).

45. Zoni, V. et al. Pre-existing bilayer stresses modulate triglyceride accumulation in the ER versus lipid droplets. Elife 10, e62886 (2021).

46. Skinner, J. R. et al. Diacylglycerol enrichment of endoplasmic reticulum or lipid droplets recruits perilipin 3/TIP47 during lipid storage and mobilization. J. Biol. Chem. 284, 30941-30948 (2009). 
47. Tauchi-Sato, K., Ozeki, S., Houjou, T., Taguchi, R. \& Fujimoto, T. The surface of lipid droplets is a phospholipid monolayer with a unique Fatty Acid composition. J. Biol. Chem. 277, 44507-44512 (2002).

48. Prattes, S. et al. Intracellular distribution and mobilization of unesterified cholesterol in adipocytes: triglyceride droplets are surrounded by cholesterol-rich ER-like surface layer structures. J. Cell Sci. 113, 2977-2989 (2000).

49. Wilfling, F. et al. Arf1/COPI machinery acts directly on lipid droplets and enables their connection to the ER for protein targeting. Elife 3, e01607 (2014).

50. Ranadive, G. N. \& Lala, A. K. Sterol-phospholipid interaction in model membranes: role of C5-C6 double bond in cholesterol. Biochem 26, 2426-2431 (1987).

51. Wenz, J. J. Predicting the effect of steroids on membrane biophysical properties based on the molecular structure. Biochim. Biophys. Acta 1818, 896-906 (2012).

52. Robalo, J. R., Ramalho, J. P., Huster, D. \& Loura, L. M. Influence of the sterol aliphatic side chain on membrane properties: a molecular dynamics study. Phys. Chem. Chem. Phys. 17, 22736-22748 (2015).

53. Almeida, P. F., Pokorny, A. \& Hinderliter, A. Thermodynamics of membrane domains. Biochim. Biophys. Acta 1720, 1-13 (2005).

54. Lin, X. \& Gorfe, A. A. Understanding membrane domain-partitioning thermodynamics of transmembrane domains with potential of mean force calculations. J. Phys. Chem. 123, 1009-1016 (2019).

55. Rog, T. \& Vattulainen, I. Cholesterol, sphingolipids, and glycolipids: What do we know about their role in raft-like membranes? Chem. Phys. Lipids 184, 82-104 (2014)

56. Bacia, K., Schwille, P. \& Kurzchalia, T. Sterol structure determines the separation of phases and the curvature of the liquid-ordered phase in model membranes. Proc. Natl Acad. Sci. USA 102, 3272-3277 (2005).

57. Grosjean, K., Mongrand, S., Beney, L., Simon-Plas, F. \& Gerbeau-Pissot, P. Differential effect of plant lipids on membrane organization: specificities of phytosphingolipids and phytosterols. J. Biol. Chem. 290, 5810-5825 (2015).

58. Choe, S. et al. The Arabidopsis dwarf1 mutant is defective in the conversion of 24-methylenecholesterol to campesterol in brassinosteroid biosynthesis. Plant Physiol. 119, 897-907 (1999).

59. Noguchi, T. et al. Brassinosteroid-insensitive dwarf mutants of Arabidopsis accumulate brassinosteroids. Plant Physiol. 121, 743-752 (1999).

60. Carland, F., Fujioka, S. \& Nelson, T. The sterol methyltransferases SMT1, SMT2, and SMT3 influence Arabidopsis development through nonbrassinosteroid products. Plant Physiol. 153, 741-756 (2010).

61. Hou, X. et al. A platform of high-density INDEL/CAPS markers for mapbased cloning in Arabidopsis. Plant J. 63, 880-888 (2010).

62. Arnqvist, L., Dutta, P. C., Jonsson, L. \& Sitbon, F. Reduction of cholesterol and glycoalkaloid levels in transgenic potato plants by overexpression of a type 1 sterol methyltransferase cDNA. Plant Physiol. 131, 1792-1799 (2003).

63. Rahier A., Benveniste P. Mass spectral identification of phytosterols. In: Analysis of Sterols and Other Biologically Significant Steroids (Nes, W. D. and Parish, E. J., eds.) (Academic Press, San Diego, 1989).

64. Fan, J., Yu, L. \& Xu, C. Dual role for autophagy in lipid metabolism in Arabidopsis. Plant Cell 31, 1598-1613 (2019).

65. Ding, Y. et al. Isolating lipid droplets from multiple species. Nat. Protoc. 8, 43-51 (2013).
66. Xu, J. et al. Cloning and characterization of an acyl-CoA-dependent diacylglycerol acyltransferase 1 (DGAT1) gene from Tropaeolum majus, and a study of the functional motifs of the DGAT protein using site-directed mutagenesis to modify enzyme activity and oil content. Plant Biotechnol. J. 6, 799-818 (2008)

\section{Acknowledgements}

This work was supported by the U.S. Department of Energy, Office of Science, Office of Basic Energy Sciences under contract number DE-SC0012704-specifically through the Physical Biosciences program of the Chemical Sciences, Geosciences and Biosciences Division. Use of the transmission electron microscope and the confocal microscope at the Center of Functional Nanomaterials was supported by the Office of Basic Energy Sciences, U.S. Department of Energy (DE-SC0012704).

\section{Author contributions}

C.X. and J.F. designed the study. L.Y., J.F. and C.Z. performed the experiments. C.X. and L.Y. drafted the manuscript, and L.Y., J.F. and C.X. revised the manuscript.

\section{Competing interests}

The authors declare no competing interests.

\section{Additional information}

Supplementary information The online version contains supplementary material available at https://doi.org/10.1038/s41467-021-25908-6.

Correspondence and requests for materials should be addressed to Changcheng Xu.

Peer review information Nature Communications thanks Hubert Schaller and the other, anonymous, reviewer(s) for their contribution to the peer review of this work. Peer reviewer reports are available.

Reprints and permission information is available at http://www.nature.com/reprints

Publisher's note Springer Nature remains neutral with regard to jurisdictional claims in published maps and institutional affiliations.

(c) (i) Open Access This article is licensed under a Creative Commons BY Attribution 4.0 International License, which permits use, sharing adaptation, distribution and reproduction in any medium or format, as long as you give appropriate credit to the original author(s) and the source, provide a link to the Creative Commons license, and indicate if changes were made. The images or other third party material in this article are included in the article's Creative Commons license, unless indicated otherwise in a credit line to the material. If material is not included in the article's Creative Commons license and your intended use is not permitted by statutory regulation or exceeds the permitted use, you will need to obtain permission directly from the copyright holder. To view a copy of this license, visit http://creativecommons.org/ licenses/by/4.0/.

(c) The Author(s) 2021 